JOURNAL DE PHYSIQUE IV

Colloque C4, supplément au Journal de Physique III, Volume 6, juillet 1996

\title{
Introduction au rayonnement synchrotron et à ses avantages
}

\author{
M. Bessière
}

LURE/CNRS-CEA-MESR, Bât. 209D, 91405 Orsay cedex, France

\begin{abstract}
Résumé
Le rayonnement synchrotron (RS) est la lumière émise par des électrons ou des positrons relativistes soumis à une accélération centripète.

Observé pour la première fois en 1947, c'est grâce aux développements technologiques de ces vingts dernières années qui ont conduit à la conception d'anneaux de stockage spécifiques à l'utilisation de ce rayonnement synchrotron (faisceaux de faibles dimensions, éléments d'insertion wigglers et onduleurs), et à la mise au point de méthodes d'investigation novatrices utilisant toutes les caractéristiques du RS (accordabilité, brillance, polarisation, faible divergence, structure temporelle), que celui-ci constitue aujourd'hui un outil scientifique de première importance pour l'étude de la matière sous toutes ses formes, dans un spectre en longueurs d'onde qui s'étend de l'infrarouge jusqu'au rayons $\mathrm{X}$.

Après une présentation rapide des caractéristiques du RS et de sa production, sa complémentarité et ses avantages par rapport aux sources classiques seront illustrés par quelques exemples d'application ayant trait aux phénomènes d'absorption, de diffraction, de diffusion et fluorescence $X$ dans les domaines de la chimie inorganique et de la physique de la matière condensée.

La possibilité, grâce à la forte brillance des machines de dernière génération, de réaliser un faisceau $\mathrm{X}$ cohérent conduisant à une nouvelle spectroscopie sera évoquée.
\end{abstract}

\section{INTRODUCTION}

L"Histoire" du rayonnement synchrotron (noté RS par la suite), même si elle semble toute récente avec le développement de son utilisation en physique, chimie, biophysique ces vingt dernières années, remonte cependant à la fin du siècle dernier avec les premiers travaux de Liénard, Wiechert et Schott sur l'émission par une particule chargée accélérée. Il a été ainsi montré que toute particule chargée rayonne du champ électromagnétique lorsqu'elle est accélérée: si cette particule est freinée (cas des électrons rencontrant l'anode d'un tube de rayons $X)$ nous avons un rayonnement de freinage connu sous le nom de Bremsstrahlung, par contre si elle est soumise à l'accélération centripète d'un champ magnétique elle émet un rayonnement électromagnétique appellé rayonnement synchrotron. 
C'est au début des années 40 que des études sont apparues sur ces rayonnements à la fois en Russie [1] et aux USA [2]. Un nouveau type d'accélérateur nommé synchrotron a ainsi vu le jour à la General Electric (USA) conduisant à la première observation du RS dans le domaine du visible [3] (pour plus de détails voir [4,5]). La théorie rendant compte de cette émission a été formulée peu après par Schwinger [6].

Le début des années 50 a vu la première ligne de RS dans le domaine ultra violet sous vide s'installer à Cornell (USA). Tomboulian, Hartman et al. [7] ont ainsi mis en évidence l'émission d'un faisceau blanc caractéristique du RS et des discontinuités d'absorption (KBe, L2,3Al). Ila fallu attendre ensuite les années 60 pour que de nouvelles études voyent le jour au près de synchrotron construit en premier lieu pour la physique de hautes énergies. Citons aus USA des études sur la polarisation (à Cornell, [8]) et sur l'absorption de gaz rares dans le domaine de l'ultra violet (au NBS à Washington, [9]), au Japon la naissance de leur premier synchrotron [10] et en Europe les travaux de pionnier autour de l'équipe de Y. Cauchois $[11,12]$ réalisés à Frascati (Italie).

C'est seuleument dans les années 70 qu'une utilisation plus intense du RS s'est précisée notamment en Europe à Hambourg (Allemagne), Daresbury (Angleterre), Orsay (France). Depuis le RS, qui n'était produit de façon parasite que sur des machines destinées à la physique des hautes énergies, s'est considérablement développé avec dans le milieu des années 80 l'apparition de machines lui étant spéciallement dédiées. La figure 1 montre de façon quasiment exhaustive les synchrotrons en fonctionnement ou en phase de construction de part le monde. Sur le sol français nous avons donc deux machines à vocation natinale, SACO dans le domaine des rayons $\mathrm{X}$ mous et du VUV et DCI dans le domaine des rayons $\mathrm{X}$, et une machine européenne (ESRF) dont le domaine d'excellence est dans les rayons $\mathrm{X}$ durs.

On notera qu'il existe aussi dans l'univers des sources naturelles de RS comme la Nébuleuse du Crabe [13].

Des méthodes scientifiques nouvelles et spécifiques, ainsi que le gain en intensité par rapport aux sources conventionnelles (qui peut dépasser $10^{6}$ ) font que le rayonnement synchrotron intéresse pratiquement toutes les disciplines scientifiques : la physique d'abord, mais aussi de façon croissante la chimie, la biologie, les sciences de la terre, l'astrophysique, la métrologie, la microélectronique ou les microfabrications, etc. D'où résulte son considérable développement depuis 20 ans; que ce soit en France ou à l'étranger.

Cet article, après une présentation rapide des caractéristiques du RS et de sa production, illustrera sa complémentarité et ses avantages par rapport aux sources classiques. Quelques exemples ayant trait aux phénomènes d'absorption, de diffraction, de diffusion et de fluorescence $\mathrm{X}$ dans les domaines de la chimie inorganique et de la physique de la matière condensée seront à cette occasion donnés. La possibilité, grâce à la forte brillance des machines de dernière génération, de réaliser un faisceau $X$ cohérent conduisant à une nouvelle spectroscopie sera évoquée.

Pour le lecteur voulant rentrer plus avant dans le fonctionnement et l'utilisation du RS, il peut consulter des ouvrages ou des articles plus spécifiques [14 à 23]

\section{QU'EST-CE QUE LE RAYONNEMENT SYNCHROTRON ?}

\subsection{Généralités et conséquences sur les machines}

La lumière émise par des particules chargées, telles que des électrons ou des positrons accélérées à une vitesse proche de celle de la lumière, est appelée rayonnement synchrotron. Le principe de base est dû à la théorie des ondes électromagnétiques de Maxwell. Cette théorie permet de calculer toutes les quantités concernant le champ électromagnétique rayonné et, bien sûr, seuls les résultats principaux seront donnés ici.

Le lecteur intéressé pourra consulter par exemple l'article original de Schwinger [6], le cours de P. Elleaume à l'école d'Aussois [16] ou le cours HERCULES [22].

La puissance rayonné par une particule suivant une trajectoire circulaire de rayon $\varrho$ est ainsi donné par la formule suivante:

$$
P=2 / 3 \mathrm{e}^{2} \mathrm{c} \beta^{4} \gamma 4 / \mathrm{e}^{2} \quad \text { (en unités CGS) }
$$

où $c$ est la vitesse de la lumière, $\beta=v / c$ (v: étant la vitesse de la particule), 


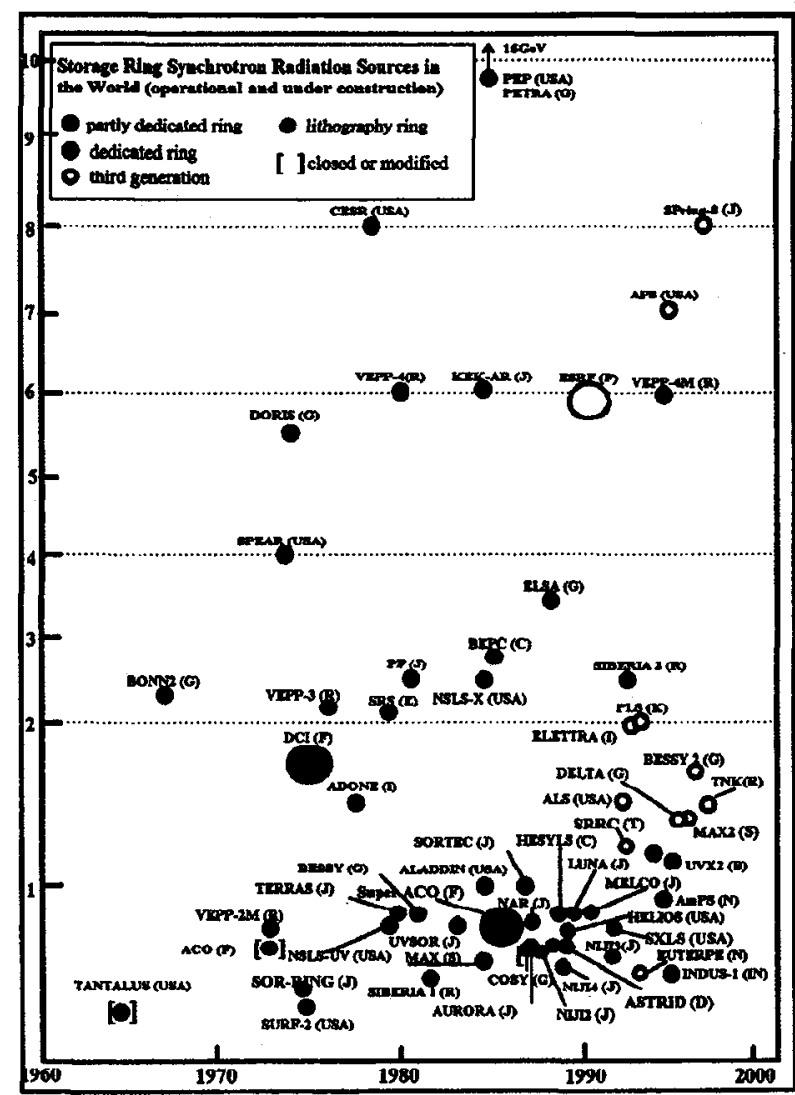

Le long de l'axe vertical l'énergie de fonctionnement de l'anneau en $\mathrm{GeV}$ est repressentative du spectre d'émission. Plus l'énergie est elevet, plus la longueur d'onde du spectre de rayonnement synchtrotron est courte.

$\begin{array}{llll}\text { Super ACO } & 800 \mathrm{MeV} & \text { VUV zayours X mous } & 500 . \mathrm{V} \\ \text { DCl } & 1.8 \mathrm{GeV} & \text { Rayons X } & 2 \mathrm{keV} \\ \text { ESRF } & 6 \mathrm{GeV} & \text { Rayons X durs } & 10 \mathrm{keV}\end{array}$

Figure 1: Anneau de stockage de rayonnement synchrotron de part le monde (document fourni par J.L. Laclare, ESRF)

$$
\begin{aligned}
& \gamma=\mathrm{E} / \mathrm{mc}^{2}=1 / \sqrt{1-\beta^{2}} \text { (E étant l' énergie de la particule et } \mathrm{mc}^{2} \text { l'énergie au repos), } \\
& \varrho=\mathrm{m} \gamma \mathrm{c} / \mathrm{eB} \text { étant le rayon de courbure de la trajectoire. }
\end{aligned}
$$

De cette simple formule on peut deja tirer les conclusions suivantes:

- plus une particule est légère, plus elle émet de RS (à énergie et rayon de courbure fixés). Le RS est un phénomène lié à l'accélération centripète dans les accélérateurs d'électrons ou de positrons. Il sera très faible dans les accélérateurs de protons ou d'ions lourds.

-seules des particules relativistes $(\beta \approx 1, \gamma>>1)$ vont rayonner une puissance conséquente. La figure 2 montre la forme de l'émission dans les deux cas limites d'une vitesse de la particule très faible, ou comparable, à la vitesse de la lumière : alors que le rayonnement est approximativement isotrope pour un particule lente, on remarque que le champ rayonné par une particule relativiste, de par l'effet de la transformation de Lorentz, est ramassé, pour une portion de trajectoire $\rho \Theta$ vue par l'observateur, dans un cône d'ouverture verticale $\Psi \approx 1 / \gamma$. 


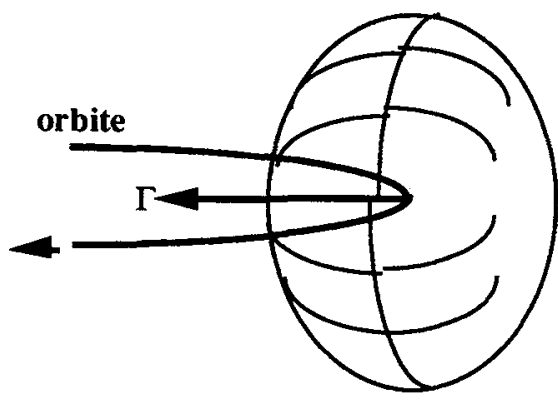

$\mathbf{v} \ll \mathbf{c}$

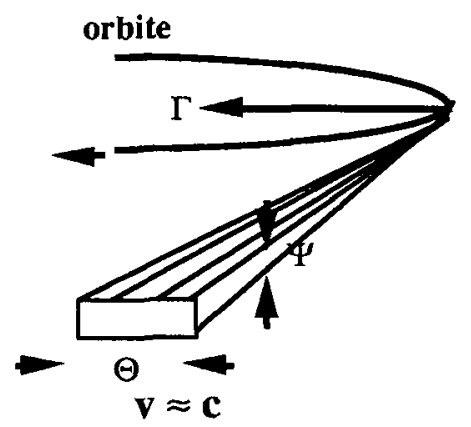

Figure 2: Emission par une particule chargé soumise à une accélération centripète.

Pour $\mathrm{N}$ particules (électrons ou positrons) concernés, on peut définír un courant équivalent I et la formule précédente donne alors la puissance rayonnée écrite dans un système d'unités appropriées:

$$
\begin{array}{lrrcc}
\mathrm{P}_{\mathrm{r}}= & 1.266 \mathrm{E}^{2} & \mathrm{~B}^{2} & \mathrm{I} & \mathrm{L} \\
{[\mathrm{kw}]} & {[\mathrm{GeV}]} & {[\mathrm{T}]} & {[\mathrm{A}]} & {[\mathrm{m}]}
\end{array}
$$

On remarquera que la puissance stockée dans le faisceau vaut quant à elle:

$$
\begin{aligned}
& P_{f}=1000 \mathrm{E} \quad I \\
& {[\mathrm{MW}] \quad[\mathrm{GeV}]}
\end{aligned}
$$

Si on applique ces formules au cas par exemple de la machine ESRF on obtient une puissance stockée de $500 \mathrm{MW}$ (soit une demi-centrale nucléaire) pour une puissance rayonnée de $460 \mathrm{~kW}$. Heureusement (!!) la puissance électrique nécessaire (6MW, essentiellement dépensée dans les éléments magnétiques) est bien inférieure à $P_{f}$ car les particules stockées conservent leur énergie. La perte principale est dû à l'émission de RS qui doit être compensée à chaque tour (rôle de la cavité HF, voir ci-après). Par contre on tomberait dans cette situation si on voulait produire le RS à partir d'un accélérateur linéaire par déviation du faisceau de particules, d'où l'utilisation pour la production de RS d'anneau de stockage dont nous allons maintenant donner les principales caractéristiques.

\subsection{Description rapide d'un anneau de stockage}

Bien que tout accélérateur d'électrons ou de positrons soit une source de rayonnement synchrotron, l'utilisation d'un anneau de stockage, machine à l'origine destinée à des fins de physique des hautes énergies, s'est imposée rapidement car, par essence, l'énergie des particules et la stabilité de leur trajectoire y est maintenue constante.

Nous prendrons comme exemple l'anneau de stockage Super-ACO de LURE (vue schématique figure 3). C'est l'une des premières machines destinée à la production exclusive de RS qui est de plus le premier exemple de machine de la "troisième génération" pouvant accepter dans les sections droites des "éléments d'insertion" dont l'intérêt et les performances seront présentés dans le paragraphe suivant. Par comparaison, les valeurs correspondantes de l'ESRF sont parfois indiquées. 


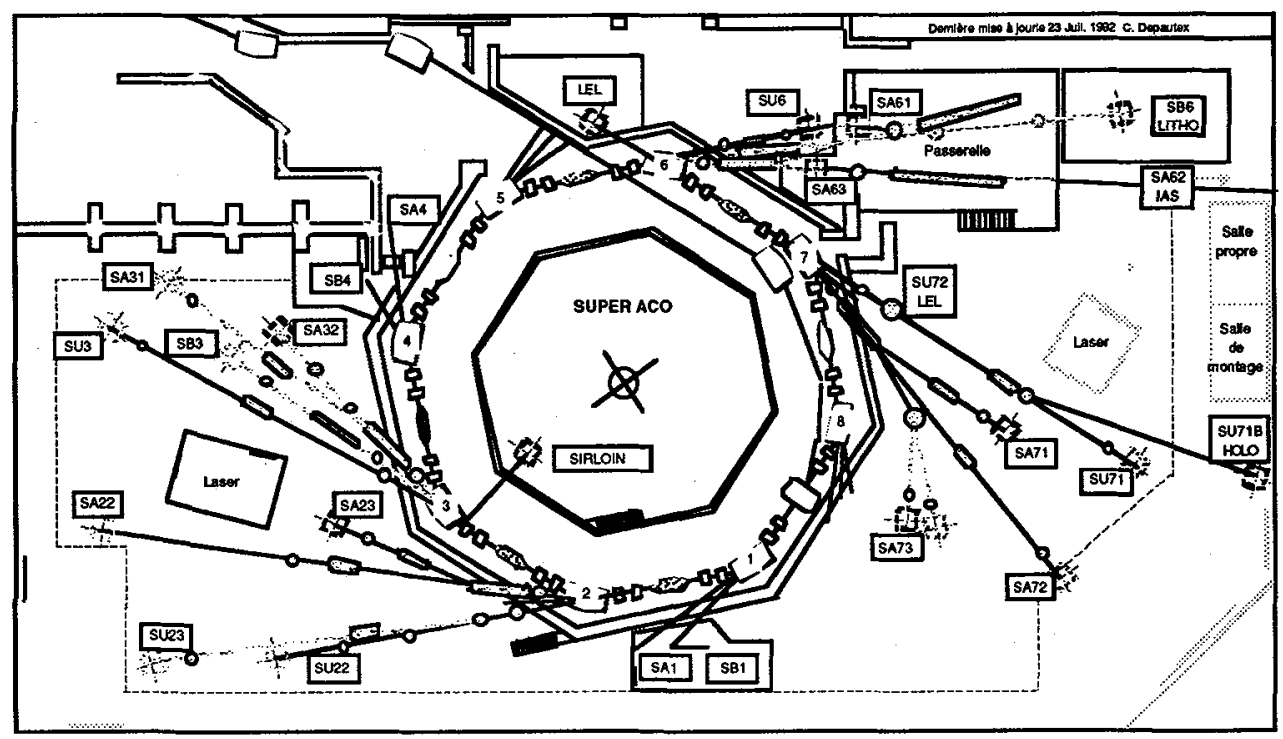

Figure 3 : Vue schématique de l'anneau de stockage Super-ACO

Passons en revue les composants principaux d'un anneau de stockage:

- Chambre à vide.

C'est à l'intérieur de cette chambre que les particules circulent. Le vide y est très poussé (quelques $10^{-10}$ Torr) pour minimiser l'interaction du faisceau de particules avec le milieu qui l'entoure. Cette interaction, plus forte pour des électrons que pour des positrons, a amené les ingénieurs de LURE à utiliser des positrons avec pour conséquence une amélioration considérable de la durée de vie. Cette solution est moins déterminante pour des machines de haute énergie comme l'ESRF $(6 \mathrm{GeV})$. Les dimensions transverses d'une chambre à vide sont par exemple pour Super-ACO 2 à $3 \mathrm{~cm}$ en hauteur et 10 à $20 \mathrm{~cm}$ en largeur. Sa longueur totale correspond à la longueur de la machine et dépend de l'énergie de cette dernière: $72 \mathrm{~m}$ pour Super-ACO, presque $1 \mathrm{Km}$ pour ESRF. Elle est refroidie sur sa partie externe pour absorber la puissance synchrotron non utilisée.

-Eléments magnétiques.

Au nombre de 8 sur Super-ACO (64 sur ESRF), les aimants de courbure imposent la trajectoire des particules qu'ils courbent avec un rayon de $1.7 \mathrm{~m}$. Ce sont des électroaimants, fonctionnant à la valeur de saturation du fer $(1.5 \mathrm{~T})$ et alimentés en série avec une stabilisation de quelques $10^{-5}$ pour des courants de quelques centaines d'Ampères. La focalisation du faisceau de particules est elle assurée par un ensemble d'éléments multipolaires (quadrupoles, hexapoles), eux aussi alimentés avec la même stabilité. L'ensemble des éléments magnétiques constitue la "maille" de la machine et se répète avec une symétrie 8 pour Super-ACO.

Comme nous l'avons déjà signalé ce sont les éléments magnétiques qui sont responsables de la majeure partie de la puissance électrique consommée, environ $1 \mathrm{MW}$ à Super-ACO pour $10 \mathrm{~kW}$ de rayonnement synchrotron fourni.

-Cavité radio-fréquence.

Son rôle est de redonner à chaque tour au faisceau de particules, par application d'un champ longitudinal périodique de plusieurs dizaines de $\mathrm{kV}$, l'énergie que celui-ci a perdu par émission synchrotron. Sa fréquence est, bien sûr, une harmonique de la fréquence de révolution des particules. Accordée en phase sur la trajectoire des particules ayant l'énergie nominale $\mathrm{E}_{0}$, elle va imposer une tension superieure aux particules plus lentes qui ont donc une trajectoire plus longue et inversement. C'est elle qui détermine ainsi le regroupement des particules en paquets : sur Super-ACO, sa fréquence de $100 \mathrm{MHz}$ compte tenu de la longueur de la machine, autorise un remplissage maximum par 24 paquets indépendants (cette valeur est de 992 sur ESRF pour une fréquence de $350 \mathrm{MHz}$ ). Elle est située sur l'une des sections droites de la machine.

L'efficacité de la cavité étant inférieure à 1 , la puissance qu'elle consomme est bien supérieure aux 10 $\mathrm{kW}$ rayonnés par émission synchrotron dans Super-ACO. 
-Dispositif d'injection.

A LURE, les machines sont injectées à partir d'un accélérateur linéaire, solution remplacée dans les installations récentes par un synchrotron de faible diamètre (booster). Les paquets stockés sont progressivement remplis par les bouffées de particules produités par l'injecteur grâce à un ensemble d'éléments magnétiques pulsés. Une fois l'intensité (le nombre de particules chargées e- ou e+ par paquet) désirée atteinte, l'injection est stoppée et l'ensemble des éléments magnétiques de l'anneau amenés à leur point de fonctionnement.

Remarque:

Le mouvement des particules le long de leur orbite n'est pas parfaitement stable, mais est affecté d'une part par les imperfections du circuit magnétique, d'autre part par l'émission de photons. Des oscillations transverses (ou oscillations betatron) sont responsables des tailles transverses du paquet et de la divergence des trajectoires, et affectent donc la brillance de la source de photons. Les oscillations longitudinales (oscillations synchrotron), dues aux fluctuations de l'énergie des particules, affecte la longueur des paquets et donc la durée des pulses de lumière. Leur comportement est bien modélisé mais sort du cadre de cet article.

\subsection{Production du rayonnement synchrotron: ses caractéristiques}

Le RS produit initiallement uniquement à partir des aimants de courbure (machine de première génération tel DCI en Fance) et maintenant fournit aussi à l'aide d'éléments d'insertion (wigglers ou onduleurs) situés dans les parties droites de l'anneau qui, déjà présents dans les machines de deuxième génération tel Super-ACO, se sont généralisés dans les machines de troisième génération tel l'ESRF.

Revenons d'abord sur les caractéristiques du RS sortant d'un aimant de courbure avant de donner quelques explications simples sur le fonctionnement et l'utilisation des éléments d'insertion.

- Aimant de courbure:

Le champ magnétique produit par les aimants de courbure induit une forte accélération centripète aux électrons (ou positrons) circulant dans la chambre à vide ayant pour conséquence, d'après ce que nous avons vue au paragraphe 1.1, l'émission de RS dans un plan tangentiel à la trajectoire des particules (figure 2).

Une première propriété remarquable est l'émission d'un spectre continu (faisceau blanc) résultant de la superposition d'un très grand nombre d'harmoniques à cause de l'effet relativiste du phénomène. La figure 4 montre la courbe universelle du spectre de RS produit par des électrons (ou positrons) d'un gigaélectronvolt.

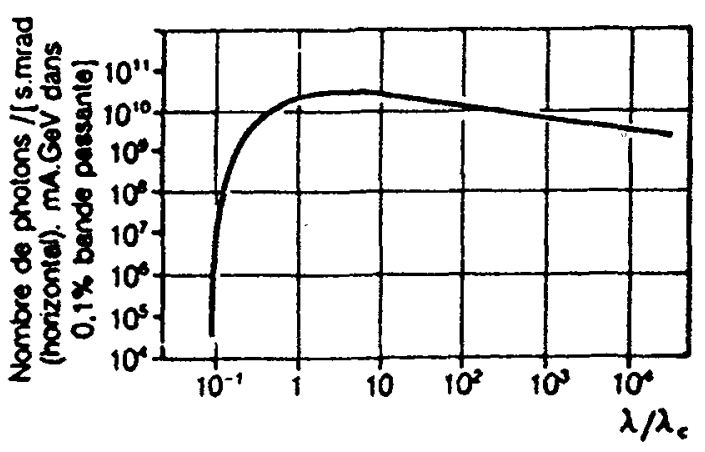

Figure 4 : Courbe universelle du spectre de RS produit par des électrons (positrons) d' $1 \mathrm{GeV}$

On définit une telle courbe par sa longueur d'onde critique qui, pour une énergie donnée des électrons, sépare en deux parties égales la puissance émise:

$$
\begin{aligned}
& \lambda_{c}=18.64 / \mathrm{B} \mathrm{E}^{2} \\
& {[\AA] \quad[\mathrm{T}][\mathrm{GeV}]}
\end{aligned}
$$


Cette formule montre que plus l'énergie est élevée, plus le spectre de longueur d'onde est déplacé vers les courtes longueurs d'ondes. Ainsi pour avoir des longueurs d'ondes dans le domaine des rayons $\mathrm{X}(0.5$ à $2.5 \AA)$ il faut que l'énergie de la machine soit supérieure à 1.5 $\mathrm{GeV}$. Pratiquement on utilise les photons jusqu'à une longueur d'onde correspondant à $\lambda_{c} / 6$. Une deuxième propriété importante est la très faible divergence de l'émission dans le plan verticale ( $\Psi=0.1$ à $1 \mathrm{mrad}$ ) conduisant à un faisceau pratiquement parallèle dans ce plan.

Cette émission de RS est aussi fortement polarisé linéairement dans le plan de l'orbite des électrons. De part et d'autre de ce plan, le taux de polarisation linéaire diminue fortement et la lumière prélevée est alors polarisé circulairement.

Enfin dernière propriété remarquable les électrons étant groupés en paquets dans la machine, ils conduisent à une émission de lumière pulsée avec des largeurs d'impulsion dans le domaine de la nanoseconde à la picoseconde ainsi qu'à des taux de répétition de quelques mégahertz.

Quelques valeurs caractéristiques définies précédemment sont données dans le tableau 1 pour les trois anneaux pris comme référence.

Tableau 1: Quelques caractéristiques d'anneaux de stockage pris comme exemple

\begin{tabular}{|c|c|c|c|}
\cline { 2 - 4 } \multicolumn{1}{c|}{} & $\lambda_{\mathrm{c}}(\AA) / \mathrm{E}_{\mathrm{c}}(\mathrm{keV})$ & Energie $(\mathrm{MeV})$ & $\rho(\mathrm{m})$ \\
\hline Super-ACO & $19 / .65$ & 0.8 & 1.8 \\
\hline DCI & $3.5 / 3.4$ & 1.8 & 3.7 \\
\hline ESRF & $.5 / 24$ & 6 & 20 \\
\hline
\end{tabular}

Eléments d'insertion:

Les éléments d'insertion en général, installés sur les sections droites de la machine, sont une suite de dipoles magnétiques alternés qui vont imposer au faisceau une trajectoire en général sinusoidale. Localement le champ magnétique appliqué et le rayon de courbure sont différents de ceux des aimants dipolaires ce qui conduit à une augmentation du flux qui vient aussi du nombre de poles utilisés.

Deux situations sont possibles pour ces éléments d'insertion suivant la valeur de la déviation angulaire maximum de la sinusoïde par rapport à la divergence naturelle du flux de photons $(\Psi=1 / \gamma)$. Le paramètre $\mathrm{K}$ définit ci-après permet de distinguer ces deux situations (onduleur et wiggler) qui sont illustrées dans la figure 5:

$$
\mathrm{K}=0.934 \lambda_{\mathrm{u}}(\mathrm{cm}) \mathrm{B}_{0}(\mathrm{~T})
$$

$\lambda_{\mathrm{u}}$ étant la période des éléments magnétiques et $\mathrm{B}_{0}$ leur champ magnétique.

\section{Onduleur}
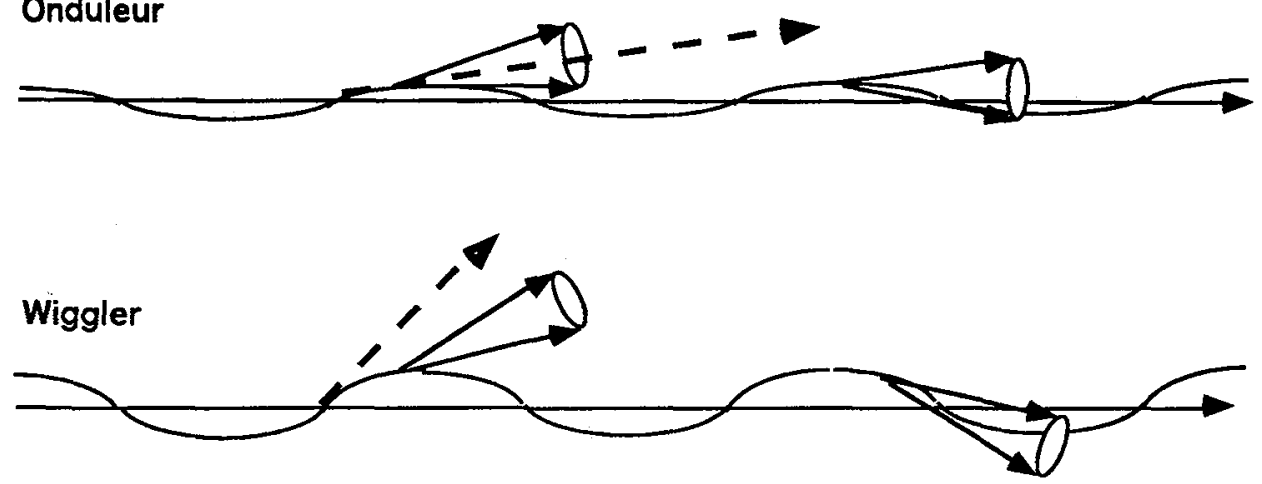

Figure 5: Trajectoire des électrons par rapport à la divergence naturelle du flux de photons pour les deux types d'éléments d'insertion 
Dans le cas du wiggler $(K>3-4)$, l'angle d'émission est supérieur à la divergence des photons et pour un observateur proche de l'axe un wiggler est un ensemble de $2 \mathrm{~N}$ ( $\mathrm{N}=$ nombre de périodes) sources indépendantes, chacune ayant les caractéristiques déterminées par le champ $\mathrm{B}_{0}$. Ce champ peut être, en utilisant des aimants supraconducteurs, très supérieur au champ dipolaire de la machine, par exemple, le wiggler supraconducteur 3 poles installe à DCI, avec un champ de $5 \mathrm{~T}, \mathrm{~K}=115$, déplace la longueur d'onde critique de 3.5 a $1.1 \AA$. D'autres solutions utilisent un nombre $\mathbf{N}$ plus grand (plusieurs dizaines) mais un champ plus faible.

Les caractéristiques d'un wiggler pour l'utilisateur sont donc un déplacement de l'énergie critique de l'anneau vers les hautes énergies en conservant un spectre blanc d'émission et un flux proportionnel à $\mathrm{N}$.

Dans le cas d'un onduleur $(K>3-4)$, il peut apparaitre des phénomènes d'interférences entre les photons émis par les différents dipoles magnétiques constituant cette élément d'insertion. Ces phénomènes d'interférence ramassent l'émission en un fondamental et ses harmoniques $\lambda \mathrm{m}$, qui dépendent de $K$, de la période $\lambda u$ du champ magnétique et de l'angle d'observation $\Theta$ sous la forme :

$$
\lambda \mathrm{m}=\left[\lambda \mathrm{u} /\left(2 \gamma^{2} \mathrm{~m}\right)\right]\left[1+\mathrm{K}^{2} / 2+\gamma^{2} \Theta^{2}\right]
$$

Dans l'axe $(\Theta=0)$ seules les harmoniques impaires sont émises, et à angle d'observation donné, le nombre d'harmoniques et le spectre en fréquence reçu varie avec $K$. Pour obtenir un fondamental de $1 \AA$, il faudrait réaliser des périodes inférieures à $\lambda \mathrm{u}=3 \mathrm{~cm}$ ce qui est techniquement difficile et nécessite une énergie de la machine de $6 \mathrm{GeV}$ (cas de l'ESRF).

Pour l'utilisateur un onduleur est donc une source de photons discontinue mais accordable en faisant varier le paramètre $\mathrm{K}$ par l'intermédiaire de l'entrefer du système magnétique. Cette source est par contre d'une extrème brillance, par exemple à l'ESRF un onduleur de $3 \mathrm{~m}$ de long à $1 \AA ̊$ présente une ouverture angulaire de $4 \mu \mathrm{rad}$ comparable à celle d'un laser. La figure 6 compare les brillances en fonction de l'énergie de différentes sources installées à l'ESRF.

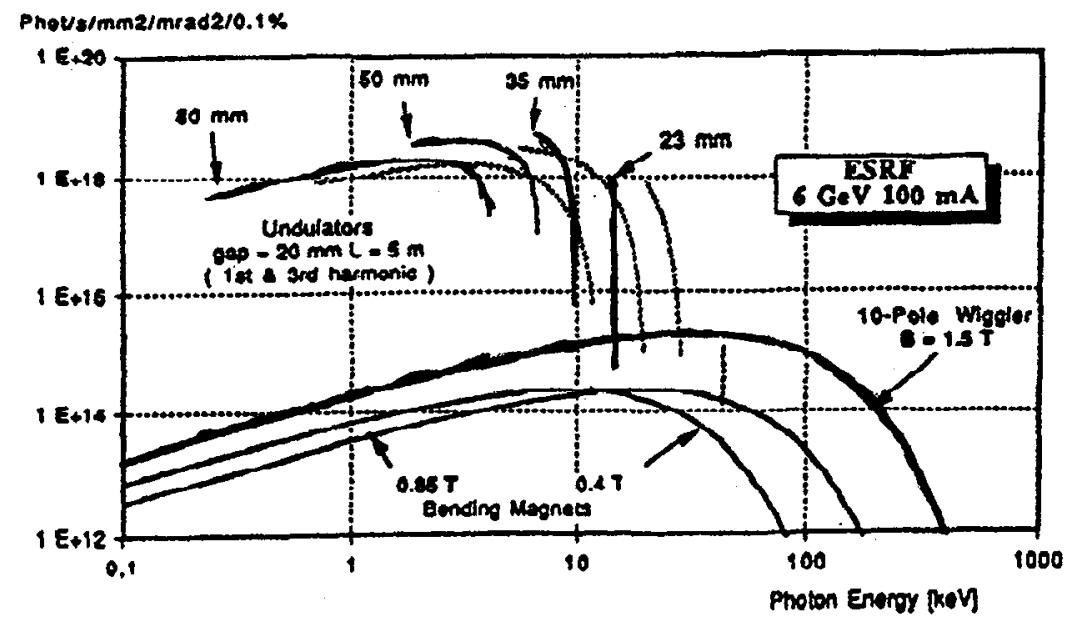

Figure 6: Brillance de différentes types de sources fonctionnant à l'ESRF (d'après rapport interne ESRF Users' Meeting 1991)

Une remarque importante sur les photons émis par les éléments d'insertion "classiques" concerne la perte de la polarisation circulaire lorsqu'on s'écarte du plan de l'orbite. En effet chaque demi-période rayonne avec une polarisation différente. Pour pallier à cette carence des systèmes plus complexes, où le champ magnétique n'est plus sinusoïdal dans un plan, sont réalisés. 


\subsection{Quelques grandeurs caractéristiques}

Pour terminer ce chapitre sur le RS et sa production il est bon de donner quelques définitions de grandeurs caractéristiques permettant de comparer les différentes sources entre elles.

D'abord définissons le flux spectral qui est la quantité d'intérêt pour les expériences "simples" du point de vue de l'optique (par exemple celle qui ne refocalise pas le faisceau sur l'échantillon). Le flux $\Phi$ est le nombre de photons $n$ émis par seconde, à travers un intervalle $\mathrm{d} \lambda$ de $0.1 \%$ de longueur d'ondes autour d'une longueur d'ondes $\lambda$, sur un arc élémentaire $\mathrm{d} \theta$ de $1 \mathrm{mrad}$ dans le plan horizontal de l'orbite et intégré dans le plan vertical:

$$
\Phi=d^{3} n /(d t d \theta d \lambda / \lambda)
$$

Ce qui donne dans le cas du flux émis par un aimant de courbure:

$$
\begin{aligned}
& \Phi=2.45710^{13} \text { I } \quad \mathrm{E} \quad \mathrm{G}(\lambda \mathrm{c} / \lambda) \\
& \mathrm{ph} / \mathrm{s} / \mathrm{mrad} \mathrm{H} / \mathrm{d} \lambda \lambda=1 \% \quad \text { [A] }[\mathrm{GeV}] \quad 1 \text { pour } \lambda \mathrm{c}=\lambda
\end{aligned}
$$

Par exemple pour $\lambda=\lambda c$ et un courant dans la machine de $200 \mathrm{~mA}$, les flux sortant d'un aimant de courbure de l'ESRF sont $2.9510^{13} \mathrm{ph} / \mathrm{s} / \mathrm{mrad}_{\mathrm{H}} / \mathrm{d} \lambda / \lambda=1 \%$, de DCI $910^{12}$, de SACO $410^{12}$.

Pour les expériences qui focalisent le faisceau sur l'échantillon, ce qui est maintenant pratiquement toujours le cas, la grandeur pertinente est la brillance dans laquelle interviennent la taille et la divergence de la source. Si $S$ est la taille de la source et $\Omega$ l'angle solide d'émission, la brillance se définit comme:

$$
\mathrm{Br}=\mathrm{d}^{6} /\left(\mathrm{dt} \mathrm{d} \Omega \mathrm{dS} \underset{\lambda}{\mathrm{d}} \frac{\lambda}{\lambda}\right) \text { soit un nombre de photons } / \mathrm{s} / \mathrm{mrad}^{2} / \mathrm{mm}^{2} / \mathrm{d} \lambda / \lambda=1 \%
$$

On remarquera que la brillance est inversement proportionnelle au produit $S^{*} \Omega$ qui doit être le plus petit possible si l'on veut une brillance élevée. Chacune des quantités $S$ et $\Omega$ se projettent individuellement aussi bien que leur produit dans les plans horizontal et vertical. On peut donc écrire:

$$
\mathbf{S} * \mathbf{\Omega}=\left(\sigma_{H^{*}}^{*} \sigma \mathrm{V}\right) *\left(\sigma^{\prime} \mathrm{H}^{*} \sigma^{\prime} \mathrm{V}\right)
$$

où $\sigma$ et $\sigma$ 'sont les écarts types de la taille de la source et de sa divergence, leurs profils étant assimilés à une Gaussienne. On a coutume de poser $\varepsilon_{\mathrm{H}}=\sigma_{\mathrm{H}} * \sigma_{\mathrm{H}}^{\prime}$ qui définit l'émittance horizontale et $\varepsilon \mathrm{V}=\sigma \mathrm{V} * \sigma^{\prime} \mathrm{V}$ qui définit l'émittance verticale. La brillance est donc maximum quand l'émittance est minimum.

Ce paramètre de brillance est maintenant courament utilisé pour comparer les sources entre elles. La figure 7 schématise l'évolution des sources de rayons $\mathrm{X}$ depuis leur découverte. Les sources de RS sont classées en trois génerations: la première correspond à des machines conçues pour la physique des hautes énergies, la deuxième génération aux premières machines construites pour l'exploitation du RS mais essentiellement constituées d'aimants de courbure comme source de photons, la troisième faisant une très grande place aux éléments d'insertion qui permettent d'obtenir les brillances les plus élevées. En 30 ans la brillance est passée de $10^{8}$ (photons $/ \mathrm{s} / \mathrm{mrad}^{2} / \mathrm{mm}^{2} / \mathrm{d} \lambda / \lambda=1 \%$ ) avec les anodes tournantes à $10^{20}$ avec les lignes onduleurs de l'ESRF.

On peut se poser la question où s'arrêtera le progrès des machines? Pour augmenter la brillance on diminue de façon générale l'émittance des électrons dans la machine mais il est inutile de viser des valeurs inférieures à la limite de diffraction (valeur indépendante de la machine et de l'ordre de $\lambda / 4 \pi$ ). Cette limite représente grossièrement la contribution des photons émis à la taille de la source vue par l'utilisateur. D'ores et déjà l'ESRF a une émittance verticale de 0.04 $\mathrm{nm}$ pour $\lambda=0.1 \mathrm{~nm}$ soit à peine 5 fois supérieure à la limite de diffraction. On peut imaginer que les machines d'une génération future viseront à atteindre cette limite de diffraction dans le plan horizontal et on peut spéculer sur une brillance optimum atteignable de l'ordre de $10^{23}$. 


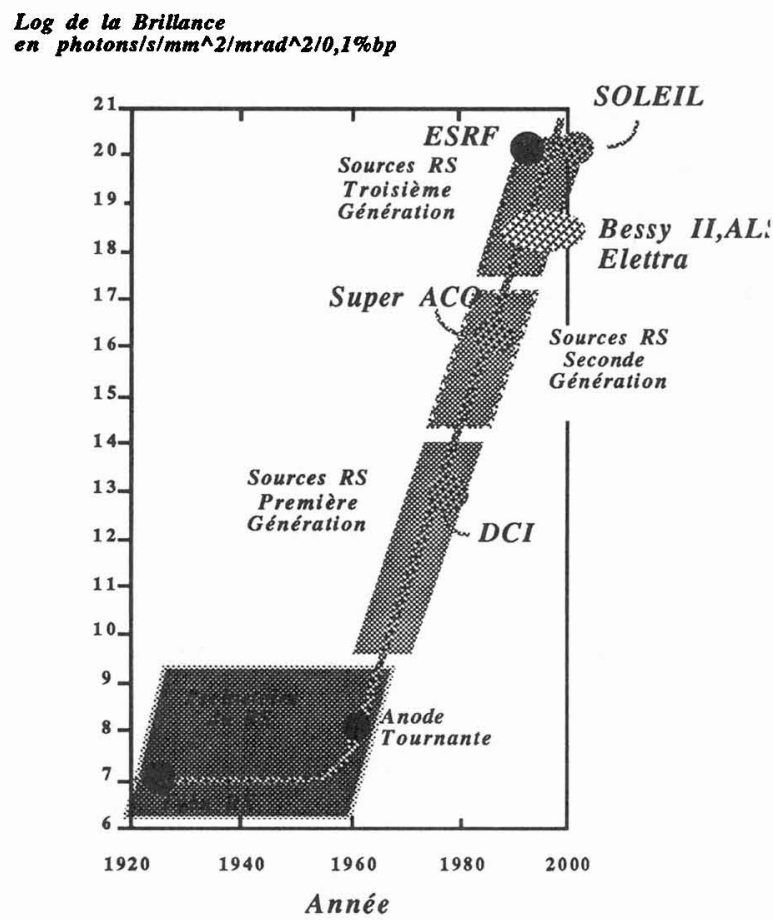

Figure 7: Evolution de la brillance des sources de RX au cours des années (document fourni par P. Thiry, LURE)

\section{PARTICULARITÉ DES STATIONS EXPÉRIMENTALES}

La nature particulière des sources de RS conduit à une instrumentation spéciallement adaptée qui diffêre en plusieurs points de celle utilisé couramment dans les laboratoires. Le premier de ces points concerne l'optique qui doit pouvoir, à partir d'un faisceau de photons émis dans un spectre continu, produire un faisceau utilisable par des techniques d'analyse comme la diffraction, l'absorption ou la fluorescence. On ne donnera ici que les grandes caractéristiques de cette optique en se limitant au cas des rayons $X$ entre typiquement 0.5 et $3 \AA$. Pour le lecteur plus curieux il peut consulter des articles de revues sur l'optique des rayons X ( cours A. Freund à l'école d'Aussois [16], [24]) ou les actes des conférences internationales sur l'instrumentation au près du RS $[25,26]$.

L'élément indispensable de cette optique est le monochromateur qui sélectionne la longueur d'onde utilisé dans le reste de l'expérience. Le principe de base dans le domaine des rayons $\mathrm{X}$ est l'application de la loi de Bragg: on utilise le plus souvent la réflexion sur des plans réticulaires (type 111 ou 220 par exemples) de cristaux parfaits de silicium ou de germanium. Ces réflexions sont en générales au nombre de une ou deux: monochromateur dit à une ou deux réflexions.

Les monochromateurs à deux réflexions peuvent être réalisés soit à partir de deux cristaux indépendants soit à partir d'un cristal monobloc (désigné souvent par l'anglicisme "channel cut"). Par exemple si on recherche à ce que le faisceau sorte à une position fixe quelque soit la longueur d'onde on choisira la solution à deux cristaux qui permet en combinant la rotation de ceux-ci avec leur écartement (mouvement de translation) d'obtenir le résultat escompté.

La puissance fournie par les photons sortant d'un élément d'insertion pouvant être conséquente (plusieurs centaines de watts) le premier cristal est alors refroidi. Si la taille du faisceau verticalement reste assez faible, il n'en est pas de même horizontalement (voir paragraphe 2.3) et il est donc utile de pouvoir focaliser le faisceau dans cette direction. Cela est obtenu en courbant sagitallement le deuxième cristal [27]. 
En ce qui concerne les mouvements de rotation il est à noter qu'une précision de la seconde d'arc est nécessaire. Enfin la dernière caractéristique de ce type de monochromateur, et général à toute l'optique auprès du RS, est la nécessité de travailler sous vide (absorption et diffusion dans l'air, problèmes de sécurité).

Les monochromateurs à une réflexion sont généralement courbes et focalisent donc en un même point de l'échantillon une large gamme de longueurs d'onde. Ils sont employés généralement pour les mesures de diffraction ou de diffusion privilégiant le haut flux par rapport à la résolution ou pour réaliser des montages dispersifs en longueurs d'onde comme dans le cas de l'EXAFS dispersif [28].

Le deuxième élément de l'optique utilisé auprès des installations de RS est constitué de miroirs basés sur le principe de la réflexion totale des rayon X et utilisés comme un filtre passe haut pour les longueurs d'onde. La réflexion totale est rendue possible pour les rayons $\mathbf{X}$ car leur indice de réfraction est 1 dans le vide et inférieur à 1 dans des matériaux comme le verre. Cette réflexion totale a lieu par contre pour des angles très faibles situés en dessous d'un angle critique qui dépend de la nature du matériau utilisé (verre simple ou recouvert d'une couche d'un élément plus lourd). Par exemple un miroir de verre avec un angle d'inclinaison de 3.5mrad ne laissera passer après réflexion que les photons de longueurs d'onde supérieures à $1 \AA$.

Ces miroirs sont généralement au nombre de deux de façon à garder les photons centrés autour du plan horizontal. Ils sont placés soit les deux après le monochromateur soit un avant et un après le monochromateur. Dans ce dernier cas, tout comme pour le monochromateur, le premier miroir est refroidi de façon à minimiser les problèmes d'échauffement du au faisceau incident. Ces miroirs peuvent aussi avoir une fonction de focalisation verticale du faisceau.

La figure 8 représente un exemple courant de configuration d'optique X [29].

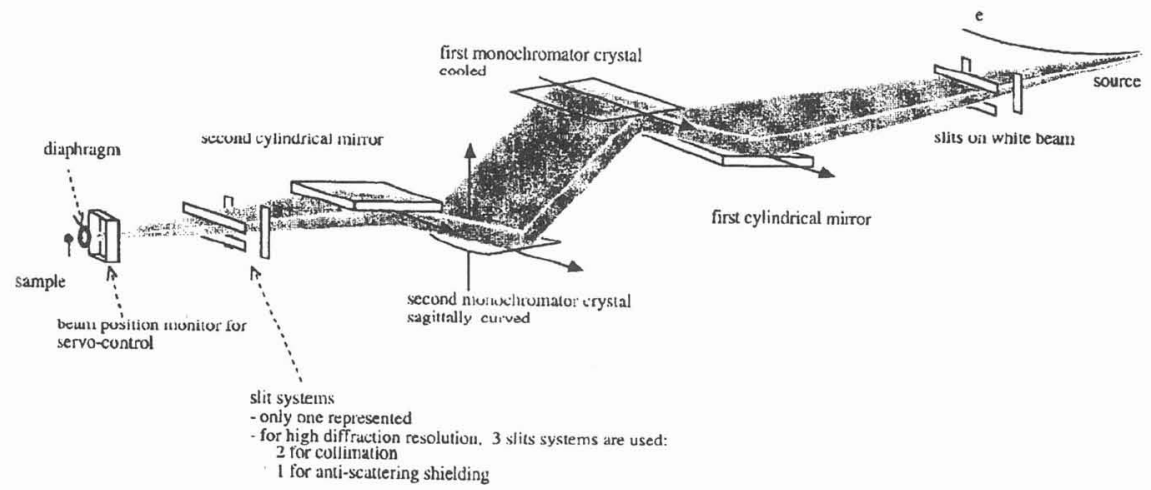

Figure 8 : Schéma optique de la ligne D2AM à l'ESRF [29]

Bien sûr dans cette description rapide il ne faut pas oublier le rôle primordiale des détecteurs à la fois pour mesurer le flux et la position du faisceau, mesures qui permettent en retour un réglage optimale.

La partie spectromètre est quant à elle très dépendante de la technique utilisée. On peut signaler que dans le cas de la diffraction de façon à tenir compte de la polarisation du faisceau dans le plan horizontale les goniomètres sont équipés de mouvement $\theta / 2 \theta$ verticaux avec une précision angulaire du millème de degré pour pouvoir réaliser des expériences de haute résolution permises grâce à la faible divergence vertical du faisceau.

\section{EXEMPLES D'ÉTUDES EN SCIENCES DES MATÉRIAUX}

On présentera ici des exemples d'études en sciences des matériaux où l'on tire profit à travers différentes techniques instrumentales des avantages du RS en montrant sa complémentarité avec les sources classiques de laboratoire. Rappelons les points forts du RS : 
- forte intensité donnant un gain de plusieurs ordres de grandeurs par rapport aux installations classiques de laboratoire

- spectre blanc d'émission

- excellente collimation du faisceau dans le plan vertical

- faisceau polarisé

- faisceau pulsé.

Il convient d'ajouter à ces caractéristiques du faisceau, sa très grande stabilité spatiale et temporelle qui permet, après l'injection des électrons dans la machine, une utilisation continue sur une échelle de plusieurs heures. Il est à noter que maintennant, grâce à des controles en boucle, la reproductibilité en position est excellente (de l'ordre de quelques microns) et que sur les machines de dernière génération le temps d'injection est très court de l'ordre de quelques minutes.

\subsection{Spectroscopie d'absorption}

Parmi les techniques structurales que le RS a promues, la spectroscopie d'absorption est un exemple typique dans la mesure où les moyens de laboratoires utilisés jusqu'alors n'en permettaient pas la pratique. Cette technique traduit les intéractions d'un électron éjecté d'une orbitale quelconque d'un atome avec les potentiels des atomes qui entourent cet élément. Pratiquement on suit cet échange en fonction de l'énergie du photon incident. On utilise donc la possibilité de choisir continuement cette énergie à l'aide d'un monochromateur et à partir du spectre blanc d'émission du RS. Le spectromètre proprement dit est constitué deux détecteurs situés avant (mesure de l'intensité incidente) et après l'échantillon (mesure de l'intensité absorbée).

Le spectre d'absorption typique donné figure 9 est la résultante d'un processus de rétroaction continu qui renferme des forces et des directions de couplage différentes entre l'onde émise et les ondes réfléchies par les diverses couches d'atomes qui entourent l'atome émetteur. Trois régions, correspondant à trois énergies différentes du photon incident, traduisent trois types de processus électroniques.

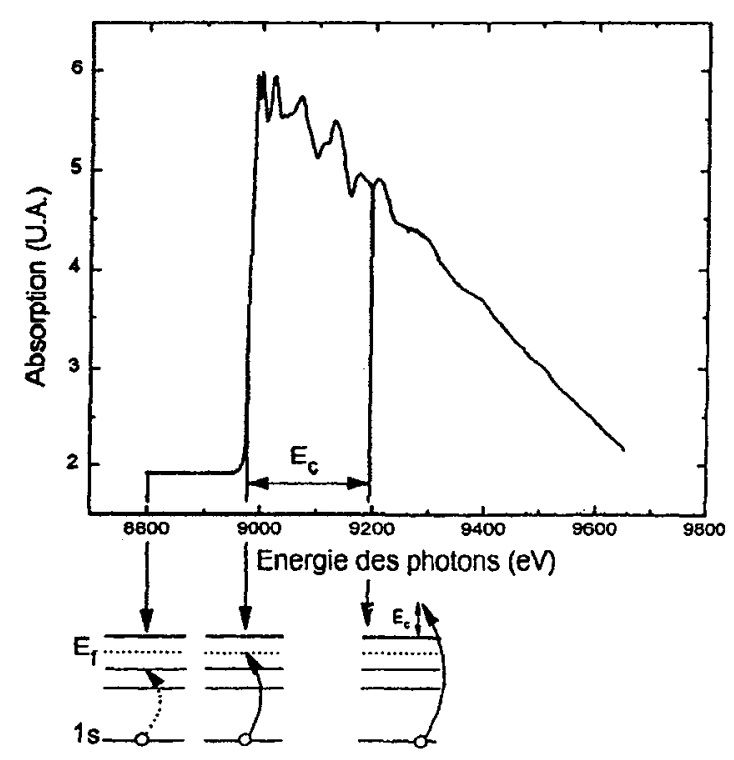

Figure 9: Spectre d'absorption d'une feuille de cuivre $\left(E_{f}\right.$ : énergie de Fermi, $E_{\mathbf{C}}$ : énergie cinétique du photoélectron éjecté)

A basse énergie la transition d'un électron à partir du niveau le plus bas n'est pas encore possible, on a une décroissance monotone de l'absorption venant des autres couches électroniques de l'atome ou des autres éléments constitutifs de l'échantillon. 
Dans un domaine d'énergie très étroit (quelques $\mathrm{eV}$ autour d'une valeur appelée seuil d'absorption, ici $8990 \mathrm{eV}$ correspondant au seuil $\mathrm{K}$ du cuivre) l'absorption augmente brutalement correspondant à l'accession possible par l'électron à des états vides juste au dessus du niveau de Fermi. Cette transition est accompagnée de structures d'absorption plus ou moins fines (prépics, pics de résonance), reflétant l'éxistence d'états inoccupés. Cette région du spectre, connue sous le nom de XANES (X-ray Absorption Near Edge Structure), est riche en informations sur la structure atomique et électronique.

Dans le domaine allant de quelques eV à quelques centaines $\mathrm{d}^{\prime} \mathrm{eV}$ au delà de ce seuil, l'absorption comporte des modulations d'amplitude décroissante : l'électron est envoyé dans le milieu avec une énergie résiduelle égale en première approximation à l'énergie du photon incident moins celle du seuil d'absorption. Cette zone est quant à elle connue sous le nom d'EXAFS (Extended X-ray Absorption Fine Structure). L'analyse de ces structures fines de l'absorption après le seuil est rendue possible grâce à une relation simple qui les lient à l'espace réel par une transformation de Fourier moyennant l'hypothèse du photoélectron libre (cours de J. Petiau, G. Krill et P. Lagarde à l'école d'Aussois [16], [30]). La distribution radiale des couches rétrodiffusantes étant représentée par le module de cette transformation mathématique du signal expérimental, on isole ainsi les divers types de liaisons et une analyse fine est alors possible couche après couche. On détermine ainsi le nombre d'atomes en premier, deuxième voir troisième et quatrième voisin (dans les cas favorables) de l'atome sondé et la distance de ces voisins à ce même atome sondé.

De nombreux exemples d'études existent dans la littérature allant de la physique ou la chimie du solide jusqu'à la biologie en passant par les catalyseurs : pour se faire une idée il n'est qu'à lire les actes de la dernière conférence internationale sur le sujet [21]. Par ailleurs on trouvera dans cet ouvrage "Rayons X et matière: 100 ans déjà" des exemples d'études par EXAFS dans le domaine des catalyseurs.

En résumé l'EXAFS est en fait un outil structural dont le succès est rapidement devenu considérable en sciences des matériaux, les raisons principales en étant :

- la spécificité en terme d'orbitale et d'éléments sondés: on peut reconstruire un arrangement atomique local à partir de la vision successivement donnée par plusieurs atomes formant le matériau

- qu'il n'est pas nécessaire d'avoir un ordre atomique étendu autour de l'élément sondé: cela permet des études sur les solides non cristallins, les milieux très divisés, les solutions, les films minces, etc..

- la possibilité d'observer directement des échantillons soumis à une contrainte chimique, mécanique, etc.

- le pouvoir de faire ces mesures dans des échelles de temps très courtes, quelques dizaines de millisecondes au mieux, et par suite d'accéder à des cinétiques de transformation de phases et des mécanismes réactionnels

- des limites de sensibilité qui peuvent être assez faibles : par exemple 0.3 at\% de Pt sur $\mathrm{Al}_{2} \mathrm{O}_{3}$.

\subsection{Diffraction et diffusion anomale}

On désigne couramment par diffraction et diffusion anomale les expériences de diffraction et de diffusion diffuse ou centrale qui utilisent les propriétés de dispersion anomale des facteurs de diffusion atomique pour les rayons X. Ce terme anomale dans le facteur de diffusion atomique est due à un phénomène de résonance entre la diffusion élastique d'une onde électromagnétique et l'absorption au voisinage d'un seuil d'absorption (absorption $\mathrm{K}$ ou $\mathrm{L}$ ).

Ce phénomène est caractéristique d'un atome donné et permet d'individualiser les atomes de structure électronique voisine. Dépendant de la possibilité de choisir la longueur d'onde et de se rapprocher du seuil d'absorption de l'atome considéré, la diffusion anomale n'a pu vraiement être utilisée qu'avec le développement du RS. Les applications sont nombreuses:

- résolution du problème de phase en cristallographie macromoléculaire et en particuliers en cristallographie des protéines. Les points principaux de ce problème très important sont résumés dans le tome II des tables intenationnales (page 266).

- caractérisation structurale des alliages présentant un ordre local ou de la démixion par diffusion diffuse anomale

- mesure des fonctions de distribution radiale dans les composés amorphes par diffusion diffuse anomale

- détermination de structures, séparation de phases ou caractérisation d'occupation de sites cristallins par diffraction anomale. 
- étude d'un site ou d'un état de valence par la structure fine d'un pic de diffraction : méthode nouvelle appelée DAFS et DANES couplant diffraction-EXAFS-XANES.

Le facteur de diffusion atomique correspond à la réponse du nuage électronique de l'atome avec l'onde électromagnétique incidente. Ce facteur de diffusion, égal au numéro atomique pour un angle de diffusion nul, varie avec le vecteur de diffusion $q=\sin (\theta) / \lambda$. On peut ici remarquer la différence avec la section efficace de diffusion des neutrons qui correspond à l'interaction neutron-noyau et qui est constant dans l'espace réciproque (très important pour la transformée de Fourier). Ce résultat simple est le cas des électrons libres. Dans le cas réel des électrons fortement liés dans l'atome, et lorsque la fréquence de l'onde incidente tend vers une fréquence de résonance correspondant à une transition électronique, il y a modification de la phase et de l'amplitude de l'onde électromagnétique de sorte que le facteur de diffusion prend la forme:

$$
f=f_{0}(q)+f^{\prime}(\omega)+i f^{\prime \prime}(\omega)
$$

fo correspond au cas des électrons libres, $\mathrm{f}$ ' et $\mathrm{f}^{\prime \prime}$ étant la signature de la diffusion "anomale" ou diffusion résonante. Ces termes $\mathbf{f}^{\prime}$ et $\mathrm{f}^{\prime \prime}$ "sont fonction de la fréquence de l'onde, c'est-à-dire de l'énergie incidente, et ils vont donc varier fortement quand on s'approche d'un seuil d'absorption (figure 10). Cette propriété est utilisée dans les expériences dites de diffraction ou diffusion anomale.

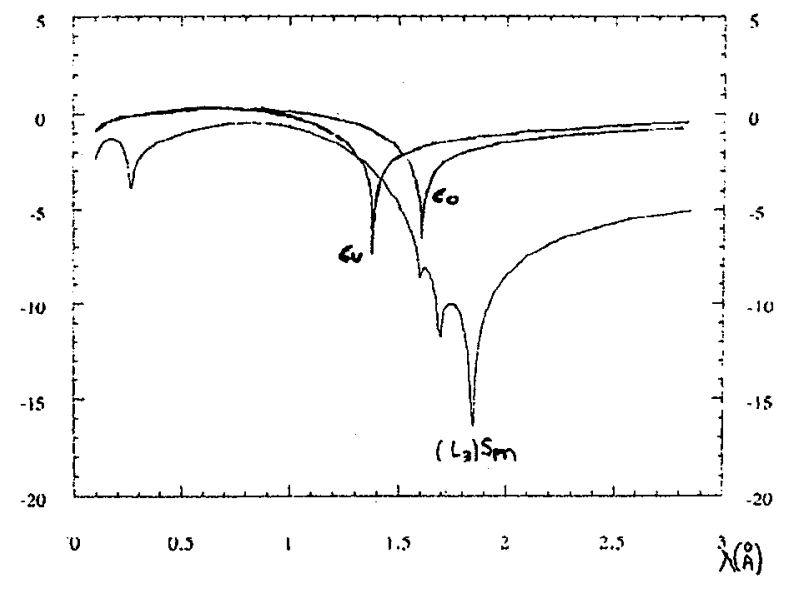

Figure 10: Exemple de variation de facteurs $\mathrm{f}^{\prime}$ aux seuils $\mathrm{K}$ du cuivre et du cobalt, au seuil $\mathrm{L}$ du samarium

Les valeurs de $\mathrm{f}$ et $\mathrm{f}^{\prime \prime}$ peuvent être calculées à partir de calculs relativistes et des tables donnant ces valeurs sont disponibles. Quand on s'approche très près du seuil $\left(\Delta \mathrm{E} / \mathrm{E}<10^{-3}\right)$ les valeurs tabulées sont mal évaluées et il est nécessaire d'effectuer leur mesures. Ces facteurs $f^{\prime}$ et $f^{\prime \prime}$ peuvent être aussi déduits soit d'une expérience d'absorption, soit par des mesures directes type interférométrie ou des mesures de l'angle de réflexion totale.

Pour donner un exemple d'application prenons le cas relativement simple de la détermination du paramètre d'ordre à grande distance dans un alliage multicomposants. Ce paramètre rend compte du taux d'occupation d'un site cristallographique par les atomes constituants l'alliage. Choisissons le cas d'un alliage de structure cubique qui s'ordonne dans la structure dite L12. Cette structure est caractérisée par deux sous réseaux: le premier en $(000)$ qui sera noté sousréseau a et les trois autres en $(1 / 21 / 20)$ (notés sous-réseaux b). Le paramètre $\eta$ d'ordre à longue distance (LRO) est défini comme la différence des proportions $a_{i}$ et $b_{i}$ de chaque élément i sur chaque sous réseau :

$\eta=a_{i}-b_{j}$, sachant que pour chaque espèce chimique, la concentration ci est telle que $c_{i}=1 / 4 a_{i}+3 / 4 b_{j}$. 
Un alliage binaire est caractérisé par un seul paramètre d'ordre obtenu par la mesure du rapport des raies dites "fondamentales" (raies d'un réseau désordonné) et les raies de "surstructure" (raies supplémentaires d'un réseau où les atomes s'ordonnent):

$$
\frac{\text { Is }}{\text { If }} \propto \frac{|F s|^{2}}{\left|F_{f}\right|^{2}}=\frac{\Sigma \eta i f i^{2}}{\mid 4 \Sigma \text { cifi }^{2}}
$$

Dans le cas d'un alliage ternaire, il y a deux paramètres d'ordre. Le rapport des intensités surstructure/fondamentale s'exprime en fonction des deux inconnues $\eta 1$ et $\eta 2$ :

$$
R(\lambda)=\frac{\left|F_{s}\right|^{2}}{\left|F_{f}\right|^{2}}=\frac{m_{1}\left(f_{1}-f_{3}\right)+\eta_{2}\left(f_{2}-\left.f_{3}\right|^{2}\right.}{\left|F_{f}\right|^{2}}
$$

Le lieu géométrique des points représentatifs est une ellipse dont l'excentricité et l'inclinaison dépendent seulement de la longueur d'onde. La détermination des deux paramètres (intersection des ellipses) nécessite au moins deux expériences (pratiquement on en utilise quatre voir plus de façon à minimiser les erreurs). Elle sera d'autant plus précise que les axes des ellipses se coupent à angle droit ce qui est obtenu en choisissant des longueurs d'onde voisine des seuils d'absorption des éléments constitutifs de l'alliage (utilisation de l'effet anomale).

Donc si dans le cas d'un alliage binaire une seule longueur d'onde suffit, la mesure pouvant être mené à bien avec un diffractomètre classique, par contre dans le cas d'alliages ternaires ou multicomposants il est nécessaire de pouvoir choisir plusieurs longueurs d'onde et donc d'avoir recours au RS.

Cette technique a par exemple été appliquée aux alliages $\mathrm{NiAlTi}, \mathrm{NiFeCr}, \mathrm{NiAlCr}$ [31] dans lesquelles les propriétés mécaniques sont dépendantes de l'état d'ordre (famille des superalliages).

Un autre exemple très voisin est la détermination de l'état d'ordre à courte distance dans un alliage ternaire. On cherche alors à obtenir le paramètre d'ordre à courte distance à travers la mesure de l'intensité diffuse dans un grand volume de l'espace réciproque. Comme précédemment dans le cas d'un alliage binaire l'étude à une seule longueur d'onde suffit tandis que pour un alliage ternaire il faut au moins trois longueurs d'onde:

$$
\begin{aligned}
& \text { pour un alliage binaire } I_{O C D}=C_{A} C_{B}\left|f_{A}-f_{B}\right|^{2} \alpha(q) A B \\
& \text { pour un alliage ternaire } I_{O C D}=C_{A} C_{B}\left|f_{A}-f_{B}\right|^{2} \alpha(q) A B+C_{C} C_{B}\left|f_{B}-f_{C}\right|^{2} \alpha(q) A B+ \\
& C_{C} C_{A}\left|f_{C}-f_{A}\right|{ }^{2} \alpha(q) C A
\end{aligned}
$$

où $\alpha(q)$ est la transformée de Fourier du paramètre d'ordre à courte distance. Pour déterminer les trois fonctions de corrélation de paire $\alpha(q)$, il faut obtenir un système d'au moins trois équations indépendantes: la diffusion anomale le permet grâce à une bonne variation de contraste (équivalent dans le principe aux mesures en diffusion neutronique avec des échantillons de composition isotopique différente). Celà a été appliqué notamment pour l'alliage (Ni3Fe).93 Cr.07 [32].

Les mesures des fonctions de distribution radiale dans les composés amorphes utilisent le même type de démarche l'intensité mesurée étant aussi une combinaison de fonctions de corrélations. Les articles sur le sujet sont nombreux, on peut consulter par exemple [33-35].

En diffusion centrale l'apport de la diffusion anomale est tout aussi important à la fois comme précédemment pour les études de systèmes multicomposants mais aussi dans le cas de composé bịnaire où des mesures à plusieurs longueurs d'onde près des seuils d'absorption des constituants permettent de séparer l'influence des défauts de celle d'une démixtion sur l'intensité mesurée [36-37]. Un point général sur ce sujet est fait dans un article de revue [38]. D'autres exemples comme l'étude des structures modulées [40] ou la diffraction magnétique résonante [41-42] pourraient être aussi donnés.

Enfin on ne peut pas terminer ce paragraphe sur l'intérêt de la diffusion anomale sans dire un mot des techniques en développement et plein d'avenir que sont le DAFS et le DANES qui combinent la diffraction avec l'EXAFS et le XANES.

Le principe en est assez simple: on suit l'évolution de l'intensité d'une raie de diffraction pendant le balayage en énergie du faisceau incident autour d'un seuil donné. Si cette raie est choisie de telle façon qu'elle soit sensible à un site particulier ou à un état de valence de l'atome 
dont on suit le seuil, on obtient une information sélective sur l'ordre local alors que l'EXAFS classique nous aurait donné un renseignement global.

Un exemple simple démontrant la faisabilité d'une telle technique a été donné par I.J. Pickering et al. [43]. Ils ont étudié un mélange de poudre de $\mathrm{Li}_{2} \mathrm{CO}_{3}$ et de $\mathrm{Co}_{3} \mathrm{O}_{4}$. L'oxyde $\mathrm{Co}_{3} \mathrm{O}_{4}$ de structure spinelle est constitué d'un réseau cfc d'oxygène avec deux sites interstitiels octaédriques de cation $\mathrm{Co}^{3+}$ et un site interstitiel tétraédrique de cation $\mathrm{Co}^{2+}$. Le diagramme de poudre comprend alors des raies spécifiques des sites octaédriques seuls, d'autres raies spécifiques des sites tétraédriques et enfin des raies propres à l'oxyde $\mathrm{Li}_{2} \mathrm{CO}_{3}$. En enregistrant en fonction des variations d'énergie au seuil $\mathrm{K}$ du cobalt l'intensité des raies $(-112)$ pour $\mathrm{Li}_{2} \mathrm{CO}_{3}$, (422) pour les sites tétraédriques et (222) pour les sites octaédriques, ils ont montré que le spectre classique d'EXAFS obtenu par la mesure de l'absorption était une combinaison pour $2 / 3$ due à la contribution des $\mathrm{Co}^{3+}$ en site octaédrique et $1 / 3$ à la contribution des $\mathrm{Co}^{2+}$ en site tétraédrique (figure 11 d'après [43]).

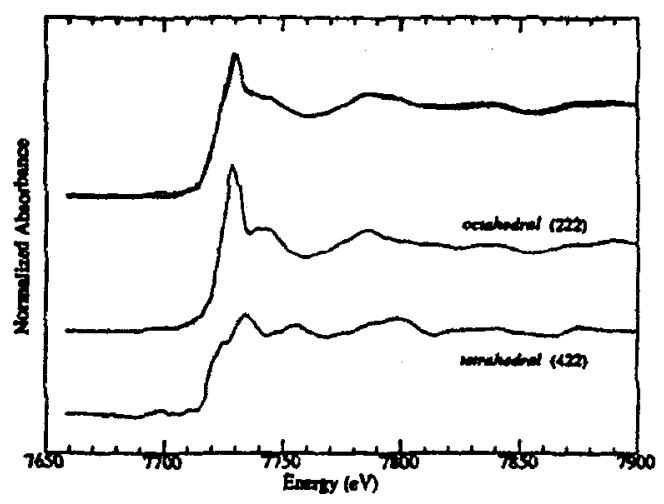

Figure 11: Seuil d'absorption au seuil $K$ du cobalt : la courbe du haut montre le signal EXAFS extrait du signal DAFS (combinaison pour $2 / 3$ du signal DAFS en site octahédrique et $1 / 3$ du signal DAFS en site tétraédrique) avec le signal d'absorption classique. Les courbes du bas montrent les signaux individuels d'absorption extraits des signaux DAFS de chaque site [43].

Les applications futures seront sans doute nombreuses comme l'étude de mélange de phases (cristallines ou amorphes), de l'état de valence d'un atome situé dans un site particulier, des multicouches (séparation coeur de la couche et interface), etc... En France cette technique se développe notamment autour de l'équipe de J.L. Hodeau [44].

\subsection{Diffraction haute résolution sur poudre et monocristal}

L'apport du RS dans ce domaine est à comparer avec ce qui est possible d'obtenir avec des montages de laboratoire. Il est bon ici de redire que les études avec le RS n'ont pas comme conséquences de supprimer celles sur des montages classiques, les deux étant très souvent complémentaires.

Les avantages dont on tire partie ici sont à la fois une largeur spectrale du faisceau incident très faible $\left(\Delta \mathrm{E} / \mathrm{E} \approx 10^{-4}\right)$ et une faible divergence angulaire dans le plan vertical qui permet d'avoir un faisceau quasiment parallèle dans ce plan. Le gain obtenu réside donc dans un élargissement instrumental minimum. Si pour les monocristaux un montage double axes (monochromateur, échantillon) apporte déja un gain substantiel, le montage le plus courament utilisé est un montage triple axes (monochromateur, échantillon, cristal analyseur) aussi bien dans le cas de la diffraction sur poudre que dans celle sur monocristal.

Pour les poudres l'utilisation d'un faisceau parallèle couplé avec une analyse des rayons diffractés selon la variable angulaire à l'aide d'un cristal monochromateur arrière supprime l'élargissement dû à l'absorption de l'échantillon ou à la divergence du faisceau [45]. Une variante consiste à remplacer le cristal analyseur arrière par des fentes de Sollers. La figure 12 
due à Parrish et al. [46] représente la même raie 111 d'une poudre de silicium enregistrée avec différents montages.

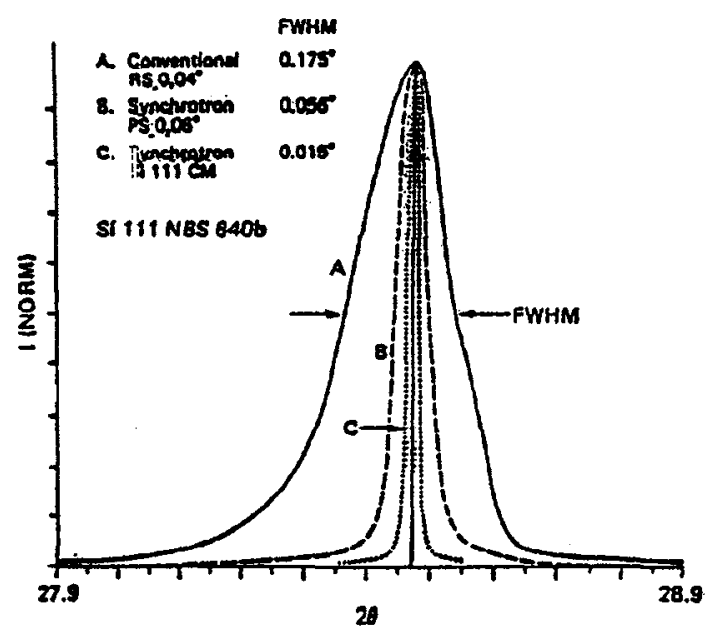

Figure 12: Comparaison du profil d'une raie 111 du silicium (NBS 640b): A) montage de laboratoire, B) montage RS avec fentes de Soller, C) montage RS avec analyseur arrière Sil11 [46].

Les applications sont nombreuses citons l'étude des profils [47], l'étude de système composé de plusieurs phases avec des raies très proches, la résolution de structures ab-initio ou encore dans des cas spécifiques pour les mesures de contraintes internes [48].

La figure 13 donne un exemple de l'apport du RS dans le cas de l'étude des phases approximantes des phases quasicristallines. Ces phases dites approximantes sont caractérisées par un diagramme de diffraction très proche de celui du quasicristal correspondant à un éclatement des raies de ce demier. Ici la haute résolution a permis de correctement identifiée un approximant orthorhombique dans le système $\mathrm{AIFeCu}$ [49].
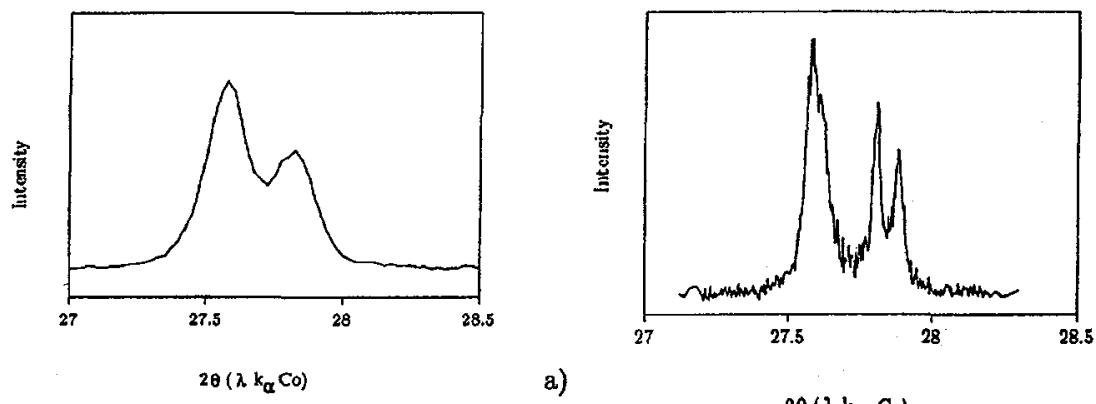

a)

$$
2 \theta\left(\lambda k_{\alpha 1} C_{0}\right)
$$

b)

Figure 13: phase orthorhombique approximante, éclatement de la raie indéxée $(6,9)$ dans la structure icosaédrique [49]: a) enregistrement sur un diffractomètre de laboratoire, b) enregistrement en haute résolution sur D23 au LURE

Pour les monocristaux ou les structures artificielles tels les superréseaux les montages type triple axes sont utilisés pour des études fines de la structure notamment des défauts. La figure 14 fournit une comparaison, pour l'étude de la structure d'un réseau de surface InP [50], entre un montage de laboratoire optimisé (monochromateur avant à 4 réflexions type DuMondBartels et analyseur arrière simple réflexion) et un montage RS haute résolution 
(monochromateur avant à 2 réflexions et analyseur arrière simple réflexion). Outre l'avantage sur la résolution, un tel montage montre aussi un gain sur le rapport signal/bruit.

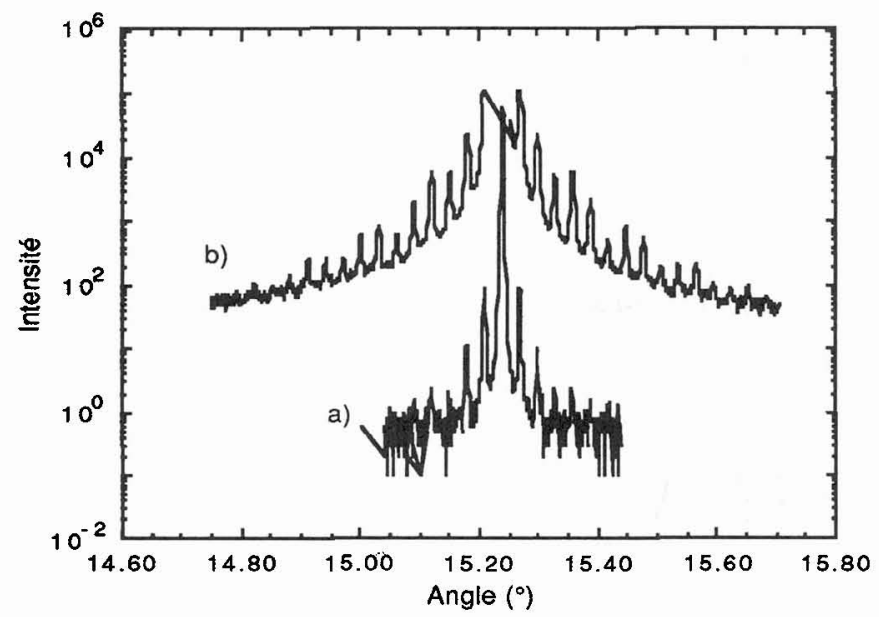

Figure 14: Comparaison d'une raie d'un réseau de surface InP et des ses raies satellites enregistrées [50]:

a) avec un montage de laboratoire haute résolution (monochromateur avant à 4 réflexions type DuMond-Bartels et analyseur arrière simple réflexion)

b) avec un montage RS haute résolution (monochromateur avant à 2 réflexions et analyseur arrière simple réflexion). La raie principale n'apparait pas car elle saturait le détecteur.

Pour donner une idée des types d'études que l'on peut réaliser citons deux exemples:

- une étude sur des monocristaux semi-conducteurs présentant des structures plus ou moins contrôlées et ordonnées (GaInAs/InP) : des cartographies (figure 15) dans l'espace réciproque permettent d'analyser de façon non destructive ces structures (réseaux de surface gravés, fils quantiques) dans les trois directions de l'espace [50].

-une étude sur un mono-grain de quasicristal (AIPdMn): l'étude entre et aux pieds des raies de Bragg sur un mono-grain recuit à $600^{\circ} \mathrm{C}$ et $800^{\circ} \mathrm{C}$ montre qu'il n'y a pas d'évidence de diffusion diffuse liée à des défauts de type "phasons", par contre un élargissement et une asymétrie du profil a été mis en évidence après recuit à $800^{\circ} \mathrm{C}$ (figure 16) qui pourraient être dues à l'introduction de contraintes internes [51].

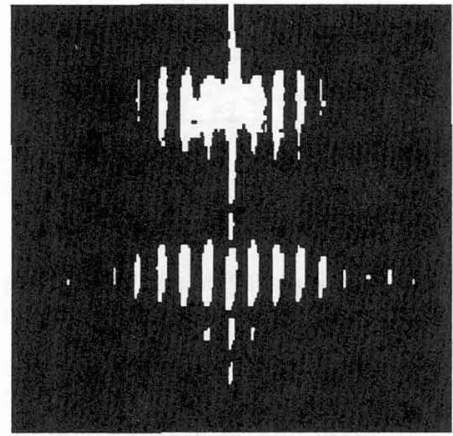

Figure 15: Cartographie d'un réseau de surface gravé dans une couche mince épitaxiale d'InGaAs/InP [50]

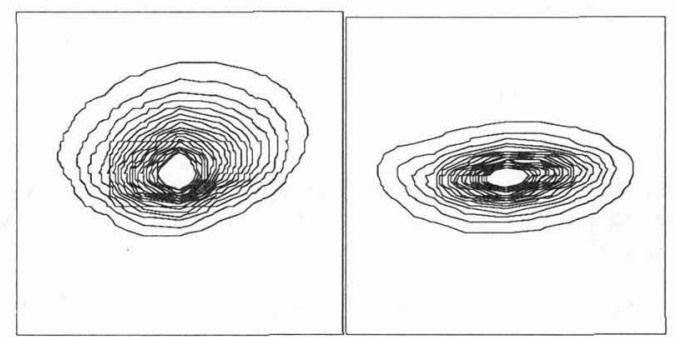

Figure 16: Evolution du pic de Bragg $(20,32)$ entre $800^{\circ} \mathrm{C}$ (à gauche) et $600^{\circ} \mathrm{C}$ (à droite) [51] 
Pour avoir un aperçu plus large sur les nouvelles possibilités offertes par le RS dans le domaine de la diffraction des poudres et des monocristaux on peut consulter l'article de J.F. Bérar et al. [52].

\subsection{Apport du RS à l'analyse et à la microanalyse $X$}

La fluorescence $X(X R F)$ est une des méthodes les plus répandues pour obtenir la composition élémentaire d'un matériau et notamment détecter et quantifier les éléments mineurs et ceux à l'état de traces. Dans cette technique, la fluorescence $X$ est en général excitée par un générateur de rayons $\mathrm{X}$ mais le RS a permis d'étendre considérablement son domaine d'application (SRXRF). Sur ce sujet un article de revue a été écrit par P. Chevallier [53], nous ne donnerons ici qu'un résumé.

Dans son principe l'analyse par fluorescence $X$ est une technique très simple. Un échantillon est placé dans une enceinte en général sous vide primaire. Un faisceau de particules irradie cet échantillon dans le but de créer des ionisations dans les couches électroniques les plus profondes des atomes le constituant. Un système de détection enregistre le spectre de fluorescence $\mathrm{X}$ émis au cours du réarrangement de ces atomes.

La diversité des faisceaux (photons, électrons, particules lourdes chargées allant du proton à l'uranium) et des systèmes d'analyse (dispersifs en longueur d'onde ou en énergie) disponibles actuellement autorise un grand nombre de combinaisons fondées sur le même principe.

Les principales caractéristiques du RS en font une source idéale pour l'éxcitation de la fluorescence $X$. De par sa faible divergence angulaire et grâce au spectre continu en énergie le RS peut être très efficacement monochromatisé sur n'importe qu'elle énergie: étant donné que la section efficace de fluorescence diminue rapidement avec l'énergie on ne perd pas beaucoup sur l'intensité des raies caractéristiques d'un élément en l'excitant avec des photons monochromatiques dont l'énergie est juste au dessus de son seuil d'absorption mais par contre on supprime pratiquement l'intensité diffusée (élastique et Compton) qui limite la sensibilité de la méthode par le fond continu qu'elle induit sous les pics caractéristiques. Ce fond continu peut être encore diminué en tenant compte de la polarisation du RS. Dans ces conditions des limites de sensibilité comprises entre 10 et $100 \mathrm{ppb}$ sont atteintes, limites bien inférieures aux autres techniques d'analyse par fluorescence $X$. La figure 17 résume ces avantages du $R S$ sur d'autres sources d'éxitations en montrant le spectre théorique de fluorescence d'un échantillon contenant $10 \mathrm{ppm}$ de divers éléments.

Un autre avantage du RS réside dans la faible énergie déposée lors du passage du projectile dans l'échantillon à étudier: à titre d'exemple pour produire une raie $\mathrm{L}_{\alpha}$ de l'or dans une feuille de 1 micron de cet élément on laisse pratiquement $1 \mathrm{MeV}$ avec des électrons de 20 à $40 \mathrm{KeV}$, $70 \mathrm{MeV}$ avec des protons de $3 \mathrm{MeV}$ et seulement $13 \mathrm{KeV}$ avec des photons de $20 \mathrm{KeV}$ !

Les exemples d'applications sont très nombreux et concernent pratiquement tous les domaines scientifiques. Les analyses auprès du RS sont réservées aux petits échantillons (submillimétriques) et à certaines études qui ne peuvent pas être réalisées avec des installations classiques, soit parce que l'échantillon est trop volumineux ou trop poreux pour être placé dans la chambre à vide (autre avantage des montages auprès du RS car ils permettent de travailler avec l'échantillon à l'air ou dans un environnement adapté) ou soit parce que l'analyse nécessite une excitation à une énergie précise. Citons quelques exemples:

- en Archéologie: le RS grâce à son faisceau collimaté et sa possibilité d'étude dans l'air est très bien adapté pour l'étude de ces échantillons aux contours très irréguliers.[54].

- en Métallurgie: utilisation de la sensibilité supérieure de l'analyse avec le RS par rapport aux autres techniques $[55,56]$ et possibilité de travailler en mini-faisceau (une centaine de microns) permettant l'étude de dendrites ou de joints de grains.

- en Astrophysique: composition élémentaire de particules cosmiques pour rechercher la (ou les) source(s) de ces échantillons extraterrestres (micrométéorites de diamètre inférieure à 100 microns) [57].

- en Géologie: analyse au niveau des éléments traces [58]. 

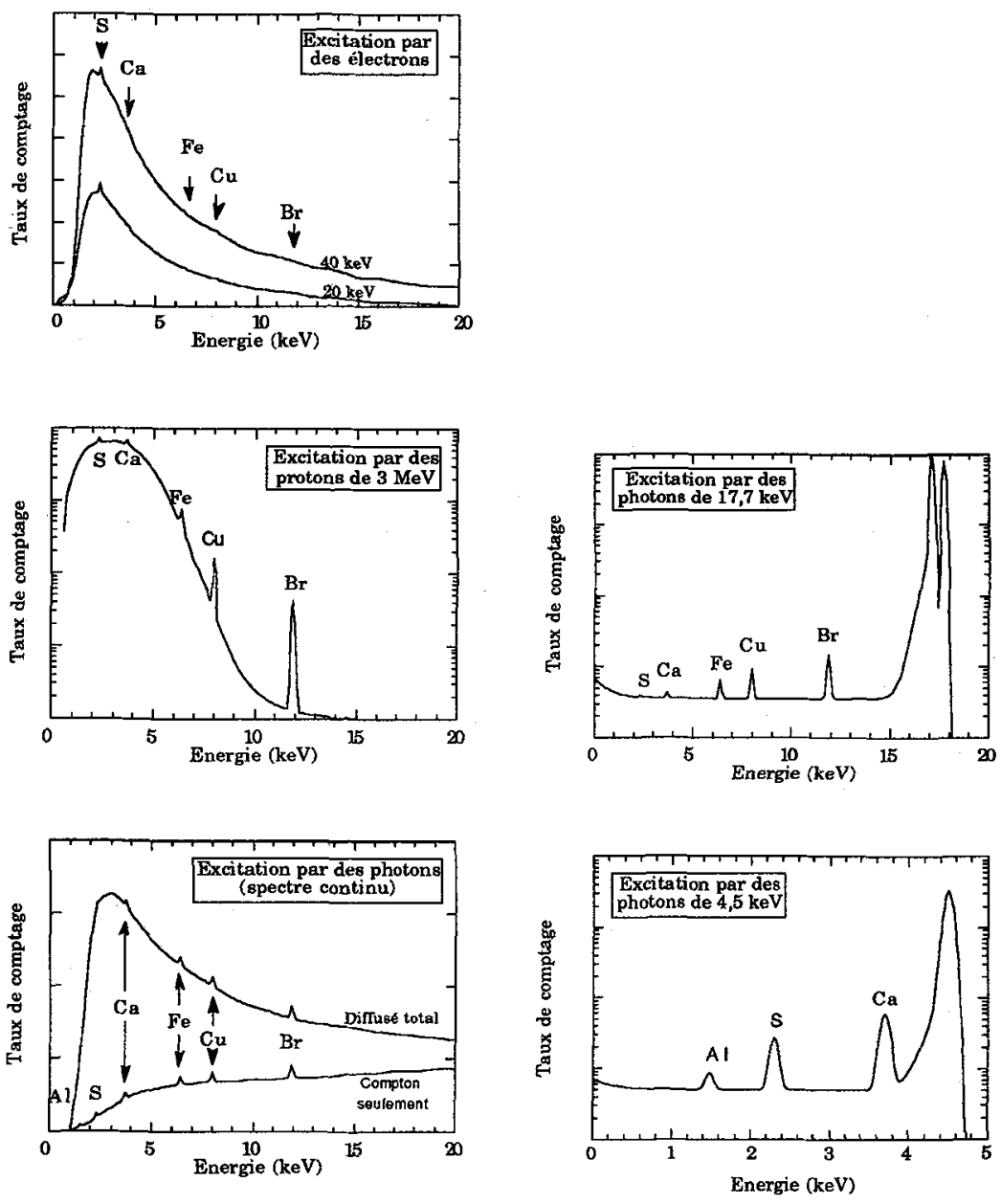

Figure 17: Spectre théorique de fluorescence d'un échantillon contenant $10 \mathrm{ppm}$ de divers éléments, excité par des électrons, des protons et des photons [53].

- en Biologie et en sciences de la vie: échantillons de grande valeur, en faible quantité, fragiles et ne pouvant en général pas être placés sous vide dans lesquels on recherche des éléments à de très faibles concentrations [59]. Par exemple la sensibilité de la méthode apparait dans la figure 18 qui représente un spectre de fluorescence $X$ d'un point de cheveu. Le spectre est obtenu en 1000 secondes et la masse de cheveu dans le faisceau est d'environ $5 \mathrm{mg}$. La concentration du fer ou du cuivre étant généralement de l'ordre de $10 \mathrm{ppm}$ cela représente moins de $50 \mathrm{pg}$ de ces éléments dans le faisceau!

Enfin la tendance actuelle est à la réalisation de microsonde photonique [60] grâce à la fois aux progrès faits en optique $X$ et à la puissance des nouvelles sources de RS. Ces progrès en optique $\mathrm{X}$ tiennent surtout à la réalisation de lentilles de Bragg-Fresnel qui associent un réflecteur de Bragg (cristal ou multicouche) et un réseau à pas variable (zone de Fresnel) pour focaliser et monochromatiser un faisceau de rayons $X$ durs avec une résolution submicronique. Elles ont été développée par Aristov [61,62] et plus tard par Erko [63] sur des multicouches et Snigirev [64] sur des cristaux. Elles sont maintenant utilisées en routine sur la microsonde photonique LURE-IMT ainsi que sur une station de l'ESRF. 


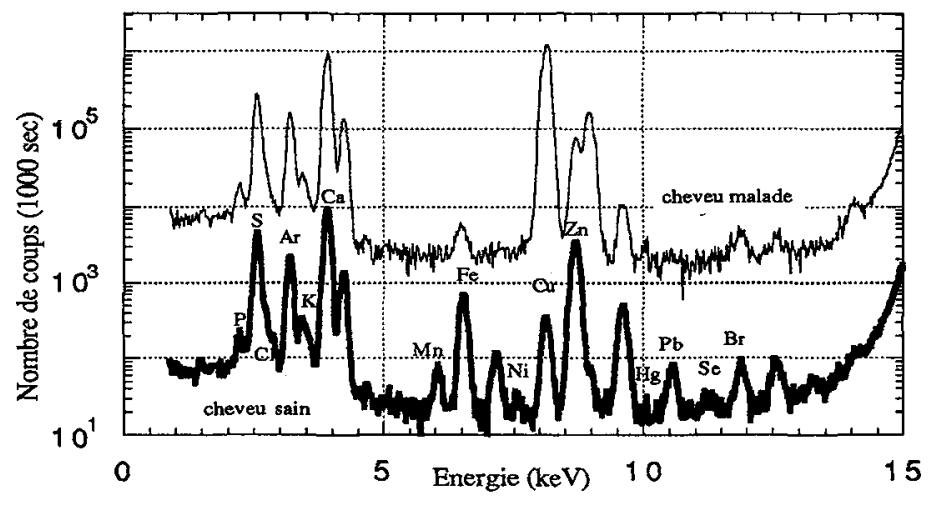

Figure 18: spectre de fluorescence X (ligne D15 de LURE) d'un point pour deux cheveux: masse de cheveu dans le faisceau est d'environ $5 \mathrm{mg}$ (document fourni par $\mathrm{P}$. Chevalier, LURE).

Sur la ligne onduleur ID10 de l'ESRF les limites actuelles (qui peuvent être améliorées d'un facteur 10) sont de l'ordre de $1 \mathrm{ppm}$ pour des éléments compris entre $\mathrm{Cr}$ et As.

Sur LURE-DCI, avec un faisceau de $50 \mathrm{~mm}$ on obtient aussi des limites de détection très intéressantes, proches de la ppm (en 1000 secondes). Bien sûr la brillance de DCI, source de première génération, est insuffisante pour permettre la réalisation de cartes de distribution pour les éléments traces. On peut cependant faire des analyses sur des points très précis d'un échantillon avec une sensibilité tout à fait suffisante. Par exemple, avec un faisceau de $12,4 \mathrm{keV}$ et de $2 \mathrm{~mm}$ de diamètre il a été enregistré un spectre de fluorescence de minuscules inclusions fluides emprisonnées dans des roches [65].

\subsection{Une nouvelle spectroscopie grâce aux $R X$ cohérents}

Ce dernier exemple représente une possibilité formidable pour l'avenir: l'utilisation de photons cohérents dans le domaine $\mathrm{X}$ pour l'étude des matériaux. C'est un des derniers challenges qu'il reste à concrétiser dans ce domaine.

Si dans le domaine de la lumière visible les lasers permettent d'obtenir un faisceau cohérent et d'étudier par exemple les fluctuations critiques près des transitions de phases dans les fluides ou la diffusion de particules dans les liquides, cela reste limité à des objets de taille supérieure à $2000 \AA$ et surtout à des matériaux non opaques. La lumière cohérente dans la région des $\mathrm{X}$ permettra elle des études de processus dynamique dans une très large gamme de matériaux allant de la métallurgie jusqu'à la matière molle en passant par les tissus vivants.

A l'heure actuelle il n'existe pas encore de lasers X mais grâce à la brillance des nouvelles sources on sait produire artificiellement à travers un trou fin situé après un monochromateur un faisceau X cohérent: la cohérence transversale (spatiale) est obtenue grâce à la taille limitée de la source tandis que la cohérence longitudinale (temporelle) vient de la faible bande passante du monochromateur.

Une des premières expériences rapportées est celle du groupe de M. Sutton [66] sur l'étude de la phase ordonnée à grande distance dans l'alliage Cu3Au: la structure en domaines ordonnés répartis au hasard, qui dans le cas d'une lumière incohérente ne produit q'un seul pic de surstructure, donne naissance ici avec une lumière cohérente à une suite de maximas reflétant les fluctuations decette structure en domaines d'orientation différente (figure 19). 


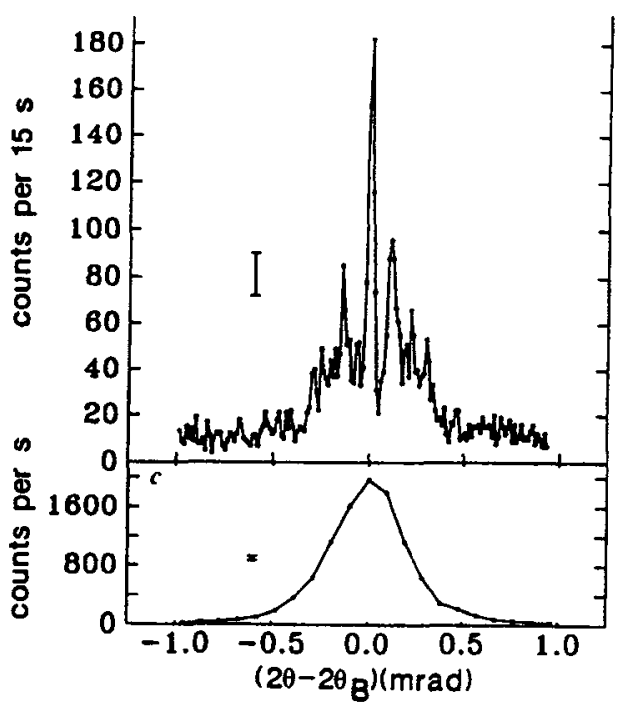

Figure 19: raie de surstructure (001) de l'alliage ordonné à grande distance $\mathrm{Cu} 3 \mathrm{Au}$, en haut en lumière cohérente et en bas en lumiere incohérente [66].

Ce type d'expérience est repris maintenant à l'ESRF notamment sur la ligne ID10 qui peut fournir un faisceau cohérent de $10^{8}$ photons/sec à $1 \AA ̊$ avec une cohérence transversale de 10 microns et longitudinale de 1 micron [67]. Quand cette lumière cohérente est diffusée par des régions distribuées au hasard (les domaines ordonnés précédents) qui introduisent des différences de phases dans cette diffussion, on observe un hologramme fait de "mouchetures" ("speckle" en anglais) qui reflète l'arrangement spatial de ces domaines [68]. On peut donc par exemple imaginer de suivre l'évolution de ceux-ci en fonction de la température. Une nouvelle spectroscopie (IFS: "Intensity Fluctuation Spectroscopy") voit ainsi le jour.

\section{CONCLUSION}

Bien que survolé rapidement et de façon partiale (seule le domaine des RX, et de plus en science des matériaux, ayant été évoqué), les différents exemples montrent la puissance d'investigation de la matière grâce au RS. On aurait pu donner des exemples tirant partie du fort flux comme les expériences de diffraction en temps réel qui permettent des enregistrements environ toutes les 50 ms [69-70] ou encore de celles utilisant le faisceau blanc comme en diffraction en dispersion d'énergie (essentiellement dans le cas d'études sous haute pression [71]. Citons aussi une technique prometteuse pour l'étude de la morphologie des agrégats la diffusion centrale sous incidence rasante [72] qui est décrite par ailleurs dans cet ouvrage [73]. Un panorama plus complet peut être trouvé par exemple dans les références [14 à 23].

Il est bien sûr évident que le RS ne résout pas tous les problèmes et qu'il reste associé dans un grand nombre de cas à d'autres techniques d'analyses. Notamment l'utilisation du RS ne signifie pas l'abandon des sources $\mathrm{X}$ classiques mais au contraire conduit à une utilisation optimale des performances de chacune.

Je rappelerai qu'en France nous possédons comme source de RS deux anneaux de stockage faisant partie du laboratoire LURE (Orsay): DCI (construit dans les années 70 et fonctionnant dans le domaine des rayons $X$ entre 3 et $50 \mathrm{Kev}$ ) et SACO (construit dans les années 80 et fonctionnant dans le domaine des rayons $\mathrm{X}$ mous en dessous de $3 \mathrm{KeV}$, de l'ultra violet et de l'infra rouge). L'ensemble offre environ 60000 heures tous instruments confondus ( 20 postes expérimentaux sur chaque anneau) représentant quelques 800 projets différents.

La France a bien sûr aussi accès au synchrotron européen installé à Grenoble (ESRF) à hauteur de $25 \%$ du temps de faisceau pour les lignes européennes et de $70 \%$ pour les lignes construites par le CNRS et le CEA (lignes CRG = Cooperative Research Group). 
Pour le début du prochain siècle il est envisagé de construire en France un nouvel anneau de stockage très performant (brillance pouvant atteindre $10^{20}$ photons $/ \mathrm{s} / \mathrm{mm}^{2} / \mathrm{mrad}^{2} / 0.1 \%$ b.p.) entièrement dédié au RS et fonctionnant entre $10 \mathrm{eV}$ et $20 \mathrm{Kev}$ (projet SOLEIL du laboratoire LURE [23]). Il serait le complément de l'ESRF vers les basses et moyennes énergies et se substituerait aux machines actuelles du LURE quirsont viellissantes.

En conclusion parmi les grands instruments les sources de rayonnement synchrotron, comme les sources de neutrons, présentent la caractéristique unique de concerner une communauté très vaste et pluridisciplinaire. Leur utilisation s'est généralisée au point d'être complètement intégrée dans la démarche scientifique de nombreuses équipes.

\section{Remerciements}

Cette introduction très générale sur le RS n'a pu être faite sans la contribution volontaire ou involontaire des "lurons", des nombreux utilisateurs de RS et bien sûr de ceux qui conçoivent et font fonctionner les anneaux de stockage. Qu'ils soient tous remerciés.

Je tiens aussi à remercier particulièrement $S$. Lefebvre, $P$. Chevalier et $P$. Lagarde qui m'ont fourni les polycopiés de leur dernier cours de Galerne dont je me suis fortement inspiré.

\section{Références}

[1] Pomeranchuk I. Ya., JETP 9 (1939) 915

[2] McMillan E., Phys. Rev. 68 (1945) 144

[3] Elder, Gurewitsch, Langmuir and Pollock, Phys. Rev. 71 (1947) 829

[4] Kulipanov G. and Skrinsky A. N., Synchrotron Radiation News V1 N3 (1988) 32-33

[5] Hartman P. L., Synchrotron Radiation News V1 N4 (1988) 32-33

[6 Schwinger J., Phys. Rev. 75 (1949) 1912

[7] Tomboulian and Hartman, Phys. Rev. 102 (1956) 1423

[8] Joos P., Phys. Rev. 4 (1960) 558

[9] Madden and Coddling, Phys. Rev. Lett. 10 (1963)

[10] Sasaki T., Synchrotron Radiation News V2 N5 (1989) 26-28

[11] Jaegle P., Synchrotron Radiation News V2 N1 (1989) 22-23

[12] Cauchois Y., Bonnelle C, Missoni G., C.R. Acad. Sci. Fr. 257 (1963) 409 et 1242

[13] Hecht E., Optics (Addison-Wesley Publishing Company, 2nd edition 1987) pp. 51-52

[14] Nenner I., Dexpert H., Bessière M,, Rayonnement synchrotron et applications, (traité Analyse Chimique et Caractérisation, Techniques de l'Ingénieur 1989)

[15] Applications du rayonnement synchrotron en Chimie, Colloque SFC 88, J. de Chimie Physique 86 N 7/8 (1989)

[16] Rayonnement Synchrotron dans le domaine des Rayons X, Ecole d'Aussois (1986), CNRS AD11 Grenoble

[17] Synchrotron Radiation Research, edited by H. Winick and S. Doniach, Plenum Press (1980)

[18] Synchrotron Radiation Techniques and Applications, edited by C. Kunz, Springer-Verlag (1979)

[20] 2nd European Conference on Progress in X-ray Synchrotron Radiation Research, edited by A. Balerna, E. Bernieri and S. Mobilio, Italian Physical Society Bologna Italy (1989)

[20] Proceedings of the First European Conference on Synchrotron Radiation in Material Research, Nuc. Inst. and Meth. in Phy. Res. B97 (1995)

[21] Proceedings of the 8th International Conference on X-ray Absorption Fine Structure, Physica B 208/209 (1995)

[22] Neutron and Synchrotron Radiation for Condensed Matter Studies, Cours HERCULES Les Editions de Physique et Springer Verlag (1994)

[23] Projet SOLEIL: argumentation scientifique, éditions de Physique (1993)

[24] Malgrange C., Acta Physica Polinica A 82 (1992) 13-32

[25] Proceedings of the 4th International Conference on SR Instrumentation, Rev. Sc. Inst. 63 (1992)

[26] Proceedings of the 5th International Conference on SR Instrumentation, Rev. Sc. Inst. 66 (1995)

[27] Elkaim E., Lefebvre S., Kahn R., Berar J.F., Lemonnier M. and Bessiere M., Rev. Sc. Inst. 63 (1992) 988-991 
[28] Dartyge E., Depautex C., Dubuisson J.M., Fontaine A., Jucha A., Leboucher P., Tourillon G., Nuc. Inns. Meth. A 246 (1986) 452

[29] Simon J.P., Geissler E., Hecht A.M., Bley F., Livet F., Roth M., Ferrer J.L., Fanchon E., Thierry JC., Rev. Sc. Inst. 63 (1992) 1051-1054

[30] Teo B.K., Basic Principles and Data Analysis dans la série "Inorganic Chemistry Concepts " édité par Springer Verlag (1986)

[31] Marty A., Bessière M., Bley F., Calvayrac Y. Lefebvre S., Acta Met. 38 (1990 ) 345-350

[32] Marty A., Cénédèse P., Bessière M., Lefebvre S., Calvayrac Y., Phys Rev. B 49 (1994) 15626-15636

[33] Fuoss P. H., Eisenberger P., Warbuton W.K., Bienenstock A., Phys. Rev. Lett. 46 (1981) 1537

[34] De Lima J. C., Raoux D., Charreire Y., Maurer M., Z. Phys. Chem. 157 (1988) 65

[35] De Lima J. C., Tonnerre J.M., Raoux D., J. Non-Cryst. Solids 106 (1988) 38

[36] Lyon O, Simon J.P., Phys.Rev B 35 (1987 ) 5164-5174

[37] Lyon O, Simon J.P., J. de Physique IV (1994) C3-25 et C3-34

[38] Simon J.P., Lyon O., Resonant Anomalous X-ray Scattering édité par Elsevier Science (1994) 305-322.

[40] Moudden A.H., Durand D., Bessière M., Lefebvre S., Phys Rev B 37 ( 1988 ) 76557661

[41] Gibbs D., Harshman D.R., Isaacs E.D., McWhan D.B., Mills D., Vettier C., Phys. Rev. Lett. 61 (1988) 1241

[42] De Bergevin F., Brunel M., Galera R.M., Vettier C., Elkaïm E., Bessiere M., Lefebvre S., Phys. Rev. B 46 (1992) $10772-10776$

[43] Pickering I.J., Sansone M., Marsch J. and George G.N., J. Am. Chem. Soc. 115 (1993) 6302-6311

[44] Vacinova J., Hodeau J.L., Wolfers P., Lauriat J.P., Elkaïm E., J. Synchrotron Rad 2 (1995) 236-244

[45] Cox D., Hasting J., Thomlinson W., Prewitt C., Nuc. Ins. Meth. 208 (1983) 315-320

[46] Parrish W., Hart M. and Toraya H., Satellite Meeting of the XVth Congress of the International Union of Cristallography (1990) 19-20

[47] Bessiere M., Quivy A., Lefebvre S., Devaud-Repski J., Calvayrac Y., J. Phys. France 1 (1991) 1823-1836

[48] Ruppersberg H., Detemple I., Krier J., Z. f. Kristallographie 195 (1991) 189

[49] Quiquandon M., Quivy A., Devaud J., Faudot F., Lefebvre S., Bessière M., Calvayrac Y., J. Phys. Condens. Matter (sous presse)

[50] Gailhanou M., Thèse Ecole Polytechnique de Lausanne (1995)

[51] Capitan M.J., Bessière M., Lefebvre S., Calvayrac Y., Quivy A. and Gratias D., Proceeding of the ICQ5 World Scientific Singapour New Jersey edited by C. Janot and R. Mossery (1995)

[52] Bérar J.F., Bessière M., Lefebvre S., J. de Chimie Physique 86 (1988) 1473-1489

[53] Chevallier P., "Projet SOLEIL: argumentaire scientifique", éditions de Physique 1993, 145-149)

[54] Chevallier P., Gruel K., Brissaud I., Tarrats-Saugnac A., Revue d'Archéométrie 17 (1993) 75

[55] Chevallier P., Abbas K., Sainfort. P., X-Ray Specrrometry 20 (1991) 292

[56] Brissaud I., Wang J. X., Chevallier. P., J. of Radioanal. and Nucl. Chem. 131 (1989) 399

[57] Chevallier P., Jehanno C., Maurette M., Sutton S., Wang J. X., J. Geophys. Res. 92E (1987) 649

[58] Chevallier P., Figueiredo M. O., Basto M.J., Abbas K., Melo Z., Ramos. M. T., X-Ray Spectrometry 22 (1993) 248

[59] Sommer F., Chevallier P., Tapiero H., Massiot P., Galle P., Silvestro L., Ariziti P.et D. Piccot. Vacuum. 42, 801, (1991)

[60] Legrand $F$., Thèse Orsay (1995)

[61] Aristov V. V., Gaponov S. V., Genkin V. M., Gorbatov Yu. A., Erko A. I., Martynov V. V., Matveeva L. A., Salaschenko. N. N., JETPh Lett. 44 (1986) 265

[62] Aristov. V. V., X-Ray Microscopy II. D. Sayre, M. Howwells, J. Kirz et H. Rarback

Edrs., Spinger series in Optical Sciences, Springer-Verlag (1988) 108

[63] Erko. A. I., J. of X-ray Sc. and Techn. 2 (1990) 297

[64] Aristov V. V., Snigirev A. A., Basov Yu. A., Nikulin Yu. A., AIP Conf. Proc. V 147 (1986) 253 
[65] Phillipot P., Chevallier P., Gibert F., Legrand F., $8^{\text {th }}$ European Union of Geosciences, Strasbourg, (Avril 1995)

[66] Sutton M., Mochrie S.G.J., Greytak T., Nagler S.E., Berman L.E., Held G.A., Stephenson G.B., Lett. to Nature 352 (1991) 608-610

[67] Brauer S., Stephenson G.B., Sutton M., Brüning R., Dufresne E., Grübel G., AlsNielsen J., Abernathy D.L., Phys. Rev. Lett. 74 (1995) 2010-2013

[68] Bley F., Livet F., Leroux J.C., Simon J.P., Abernathy D.L., Als-Nielsen J., Grübel G., Vignaud G., Dolino G., Legrand J.F., Camel D., Menguy N., Papoular M., Acta Cryst. A 51 (1995) 746-753

[69] Larson E.M., Wong J., Holt J.B., Waide P.A., Nutt G., Rupp B., Terminello L.J., $J$. Mater. Res. Vol. 8 No. 7 (1993) 1533-1541

[70] Berar J.F., Lemonnier M., Bartol F., Gramond M., Chevreul J., Nuc. Ins. Meth. B 82 (1993) 146-150

[71] Sadoc A., Itie J.P., Polian A., Lefebvre S., Bessiere M., Calvayrac Y., Phil. Mag. B 70 (1994) 855-866

[72] Naudon A., Slimani T., Goudeau P., J. Appl. Cryst. 24 (1991) 501

[73] Thiaudière D. et Naudon A., "Diffusion centrale des rayons $X$ sous incidence rasante.

Etude de la morphologie des agrégats", cet ouvrage. 